Check for updates

Cite this: Mater. Chem. Front. 2020, 4, 2825

Received 3rd June 2020,

Accepted 1st July 2020

DOI: 10.1039/d0qm00368a

rsc.li/frontiers-materials

\section{Development and advancement of rotaxane dendrimers as switchable macromolecular machines}

\author{
Chak-Shing Kwan and Ken Cham-Fai Leung (D) *
}

Rotaxane dendrimers are a newly emerging large family of mechanically interlocked molecules (MIMs), which combine the concept of molecular switching properties into hyperbranched dendrimers to render new macromolecular machines. Basically, rotaxane dendrimers are divided into three types and each type represents a special connection between the mechanical bonds, macrocycles and rods. In the past few years, with the methodology development of rotaxane synthesis, more complex and high molecular weight rotaxane dendrimers have been designed and synthesized, especially the most complicated Type III rotaxane dendrimers. In particular, higher generation sophisticated rotaxane dendrimers bring advancements from 1-dimensional to 3-dimensional molecular switching, and have been applied in advanced functional materials for various applications towards drug delivery. This review surveys the development and advancement of each type of rotaxane dendrimer, from the simple dendron-grafted Type I to intricate Type III dendritic polyrotaxane dendrimers with future perspectives.

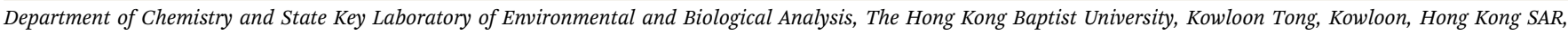
P. R. China.E-mail: cfleung@hkbu.edu.hk

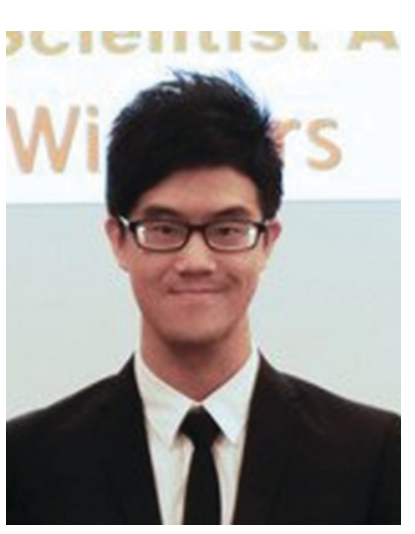

Chak-Shing Kwan
Dr Kwan received his BSc degree in 2014 and PhD degree in 2018 under the supervision of Professor Ken Cham-Fai Leung in The Hong Kong Baptist University. He was a postdoctoral researcher in The Hong Kong Polytechnic University in 2019. He is currently a postdoctoral research associate at The Advanced Science Research Center, The City University of New York, USA. In 2020, he received the Humboldt Research Fellowship for Postdoctoral Researchers. His research interests are supramolecular chemistry and polymer chemistry.

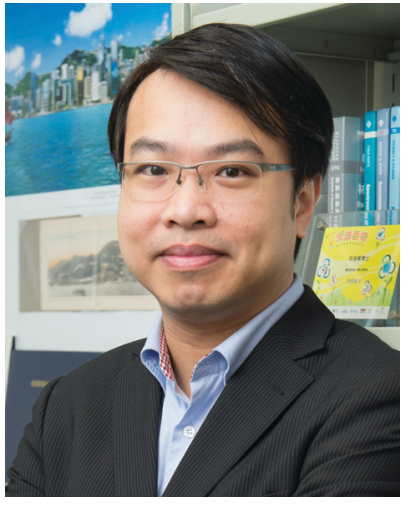

Dr Leung is an Associate Professor at the Department of Chemistry, The Hong Kong Baptist University, Hong Kong SAR, P. R. China. He concurrently holds the Honorary Associate Professorship at the Faculty of Dentistry, The University of Hong Kong. His research interests are supramolecular chemistry, catalysis, nanoscience, and nanomedicine. He gained his $B S c$ and PhD degrees in Chemistry from The Chinese University of Ken Cham-Fai Leung Hong Kong in 1999 and 2003, respectively. He conducted a three-year postdoctoral research work in the laboratory of Nobel Laureate in Chemistry, Professor Sir Fraser Stoddart in 2003-2006 at The California NanoSystems Institute and Department of Chemistry and Biochemistry, The University of California at Los Angeles, USA. He started his independent research career at The Chinese University of Hong Kong before moving to The Hong Kong Baptist University in 2012. He has published over one hundred articles in peer-reviewed international journals. 


\section{Introduction}

Dendrimers are hyperbranched macromolecules with tree-like dendritic branching units. The word dendrimer originally came from the Greek 'dendron' for 'tree' or 'branch' and 'meros' for 'parts'. Dendrimers are subsets of polymers, from which dendrimers possess unique monodispersity with well-defined semi-globular to globular architectures. Dendrimers were first reported by Vögtle et al. in $1978 .{ }^{1}$ The basic components of a dendrimer are dendrons, whereas a dendron is defined as a highly branched sector with multiple surface groups on the periphery. ${ }^{2}$ Two or more dendrons link together covalently into a multivalent core composing a dendrimer. The synthesis of dendrons/dendrimers can be mainly categorized as divergent and convergent approaches. For the divergent approach, the synthesis of dendrimers is started by building up the peripheral branching groups from the core, generation by generation. On the other hand, the convergent approach starts from the peripheral groups building to the core end point, to become a dendron segment. ${ }^{3}$ Dendrons of various generations (layers) are then coupled together to produce dendrimers of various generations. The concept of the convergent approach was first introduced by Hawker and Fréchet. ${ }^{4}$ Since hyperbranched dendrimers are theoretically spherical macromolecules with high molecular weight, the interior void phase between the branches can encapsulate or trap 'cargo' inside the macromolecules, and thus they can be used for various potential applications, ${ }^{5}$ based on varying the different branching units and the surface groups. With this interesting property, dendrimers have been widely developed and applied, for example in drug/gene delivery, ${ }^{6,7}$ nanomedicine, ${ }^{7}$ nanoparticles, ${ }^{8}$ catalysis, ${ }^{9}$ light-harvesting materials, ${ }^{10}$ and liquid crystals. ${ }^{11,12}$

Rotaxane is a member of the mechanically interlocked molecules (MIMs) that consists of a linear dumbbell-shaped component known as the 'rod' and a macrocycle component known as the 'ring'. Both ends of the rod are linked by bulky groups known as stoppers, such that one or more macrocycles can encircle the rod and be trapped between the two stoppers. The word rotaxane derives from the Latin words 'rota' and 'axis', meaning 'wheel' and 'axle', respectively. There are several strategies to synthesize a rotaxane, including 'threading followed-by stoppering', 'stoppering followed-by clipping', active metal template, ring slippage and stopper swelling. ${ }^{13,14}$ Among these approaches, the molecular recognition unit between the macrocycle and rod can be utilized by noncovalent interactions (mechanical bonds) such as hydrogen bonds, electrostatic interactions, $\pi$-stacking, etc. A pseudo-rotaxane represents an interlocked structure between a macrocycle and non-stoppered rod. The threading followed-by stoppering process is a common method for synthesizing a [2]rotaxane. A fascinating property of rotaxanes is the molecular shuttling (switching) process within the interlocking molecule. ${ }^{15}$ Since rotaxanes are maintained by the entanglement between two or more molecular entities through space, and the components cannot be separated without breaking the covalent bond, the macrocycle can be shuttled from one side to the other side on the rod upon different external stimuli such as $\mathrm{pH}$, temperature, redox,
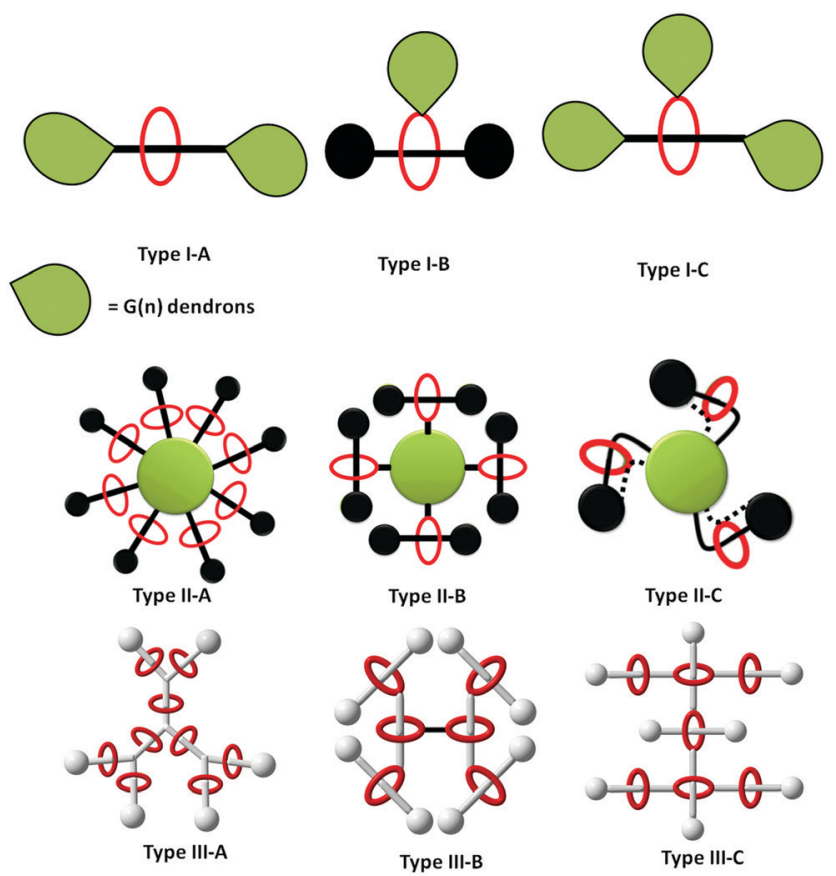

Fig. 1 Molecular structures of the Type I, II and III rotaxane dendrimer family. Type I is known as a dendron-grafted rotaxane, where dendrons (green) are grafted on the thread for I-A, on the macrocycle (red) for I-B, and on both the thread and the macrocycle for I-C. Type II refers to a rotaxane connected to the outermost branches of the dendrons or the peripheries of the dendrimers (green), where II-A is connecting with the thread, II-B is connecting with the macrocycle, and II-C is connecting with both the thread and the macrocycle (directly or indirectly). Type III is known as a dendritic polyrotaxane, where III-A refers to multiple rings threaded to a dendrimer scaffold, III-B refers to the rotaxane mechanical bonds constituted in all branching points through macrocycle-thread connections, and III-C refers to the rotaxane mechanical bonds in both the branching points and the core of the dendrimer.

light, etc. ${ }^{16}$ With the development of new synthetic strategies and new rotaxane structures, more sophisticated rotaxane molecules were prepared and used as molecular machines. ${ }^{17}$

Since both dendrimers and rotaxanes have their own characteristic properties, the combination of rotaxanes and dendrimers can generate a new class of macromolecule known as 'rotaxane dendrimers', where this type of compounds can possess the characteristics of both the rotaxane and dendrimer (Fig. 1). The term rotaxane dendrimers was first defined by Lee and Kim in $2003,{ }^{18}$ and further elaborated by Stoddart in $2016 .{ }^{19,20}$ Generally, rotaxane dendrimers can be divided into three types (I, II, III), and each type can be further sub-classified (A, B, C). In this review, we will survey (1) all up-to-date examples of rotaxane dendrimers in the past three decades, (2) all types of rotaxane dendrimers with the highest generation, and (3) switching behaviors and applications of rotaxane dendrimers.

\section{Types of rotaxane dendrimer}

\subsection{Type I rotaxane dendrimers}

Type I rotaxane dendrimers are classified as a dendron scaffold grafted onto simple (pseudo)rotaxane structures, either on the 
thread (Type I-A), macrocycles (Type I-B) or both (Type I-C). Since the concept of Type I rotaxane dendrimers is simply incorporating the dendrons onto the rotaxane core components, it is relatively synthetically accessible. Thus, Type I rotaxane dendrimer structures have been reported the earliest in the field. Fréchet-type benzyl ether dendrons are sometimes the choice of dendrons grafted onto the interlocking component as a stopper, to produce Type I rotaxane dendrimers (Fig. 2). Many more different dendrons have been grafted onto Type I rotaxane dendrimers.

2.1.1 Type I-A rotaxane dendrimers. Type I-A rotaxane dendrimers refer to the attachment of dendrons onto the rods of the rotaxane. The dendrons in Type I-A usually act as the stopper with different dendron generation to prevent the slippage of macrocycles. Stoddart et al. first reported a series of rotaxanes 1 and 2 based on the donor-acceptor interaction of bipyridinium $\left(\mathrm{BIPY}^{2+}\right)$ with bis-para-phenylene-34-crown-10 (BPP34C10) in 1996 (Fig. 2). ${ }^{21}$ The synthesis involved the self-assembly of BIPY ${ }^{2+}$ with BPP34C10, followed by the nucleophilic attack of the G3 Fréchet dendron bromide to generate a series of [2], [3] and [4]rotaxanes containing one to three rings in the structures. A temperature dependent molecular shuttling process was also demonstrated with this example.

Vögtle et al. used a trapping method as the template for synthesizing [2]rotaxane 3, stoppering with G1 to G3 Fréchet dendrons and studying the size effect of the dendrons on the deslipping kinetics (Fig. 2). ${ }^{22}$ Tetralactam macrocycles and oxymethylene dumbbells stoppered with tris(tert-butylbenzene) were used for the formation of a wheel-phenolate complex template. Their results showed no rotaxane formation with G1 Fréchet dendrons, while G2 and G3 [2]rotaxanes were stable at room temperature, but deslipped at high temperature $\left(150{ }^{\circ} \mathrm{C}\right)$.

Loeb et al. demonstrated the recognition between 1,2-bis(pyridinium)ethane (BPE) and DB24C8 as the template synthesis of [2]rotaxane 4 stoppering with G0 to G3 Fréchet dendrons. ${ }^{23}$ They investigated the solvent effect of different dendrons'
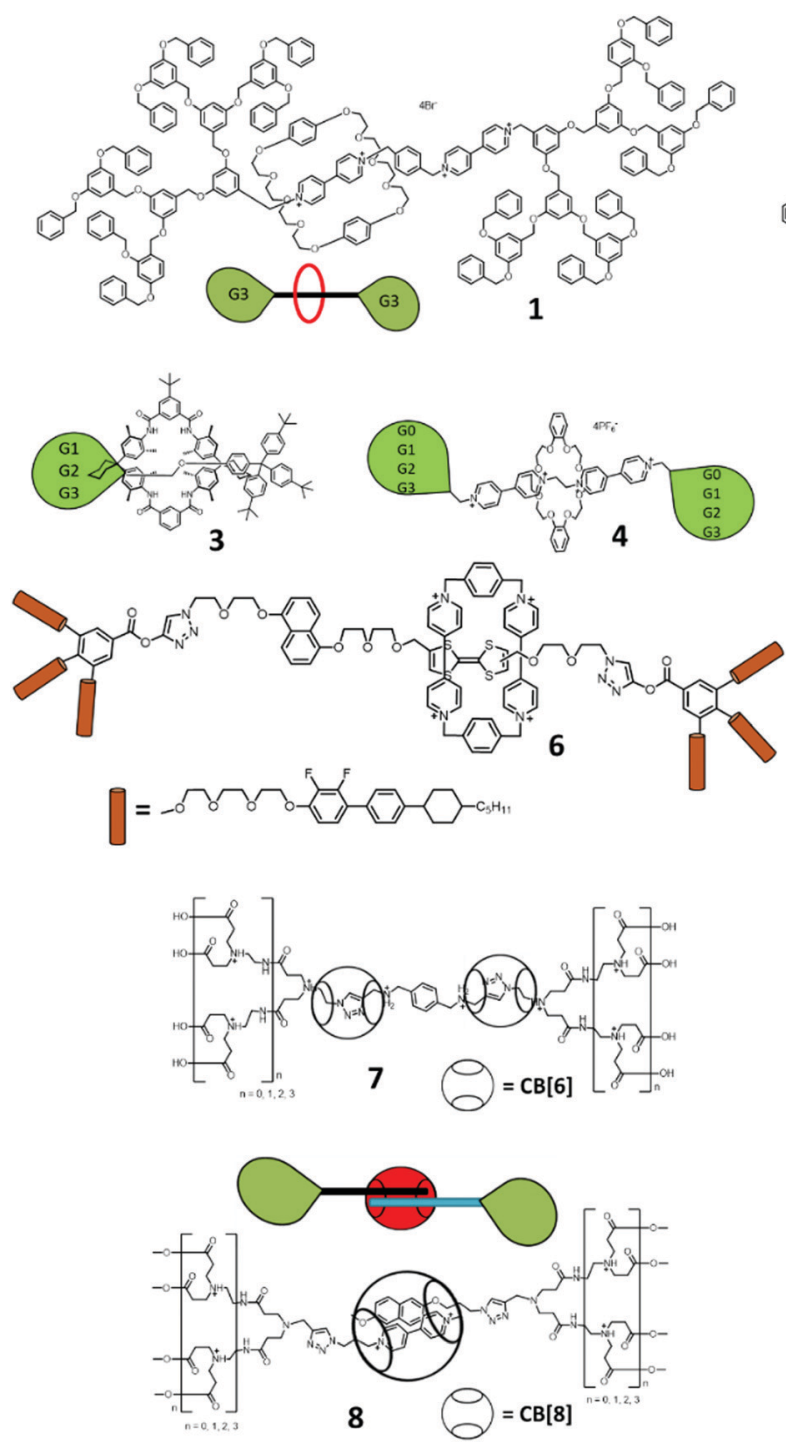

Fig. 2 Type I-A rotaxane dendrimers.
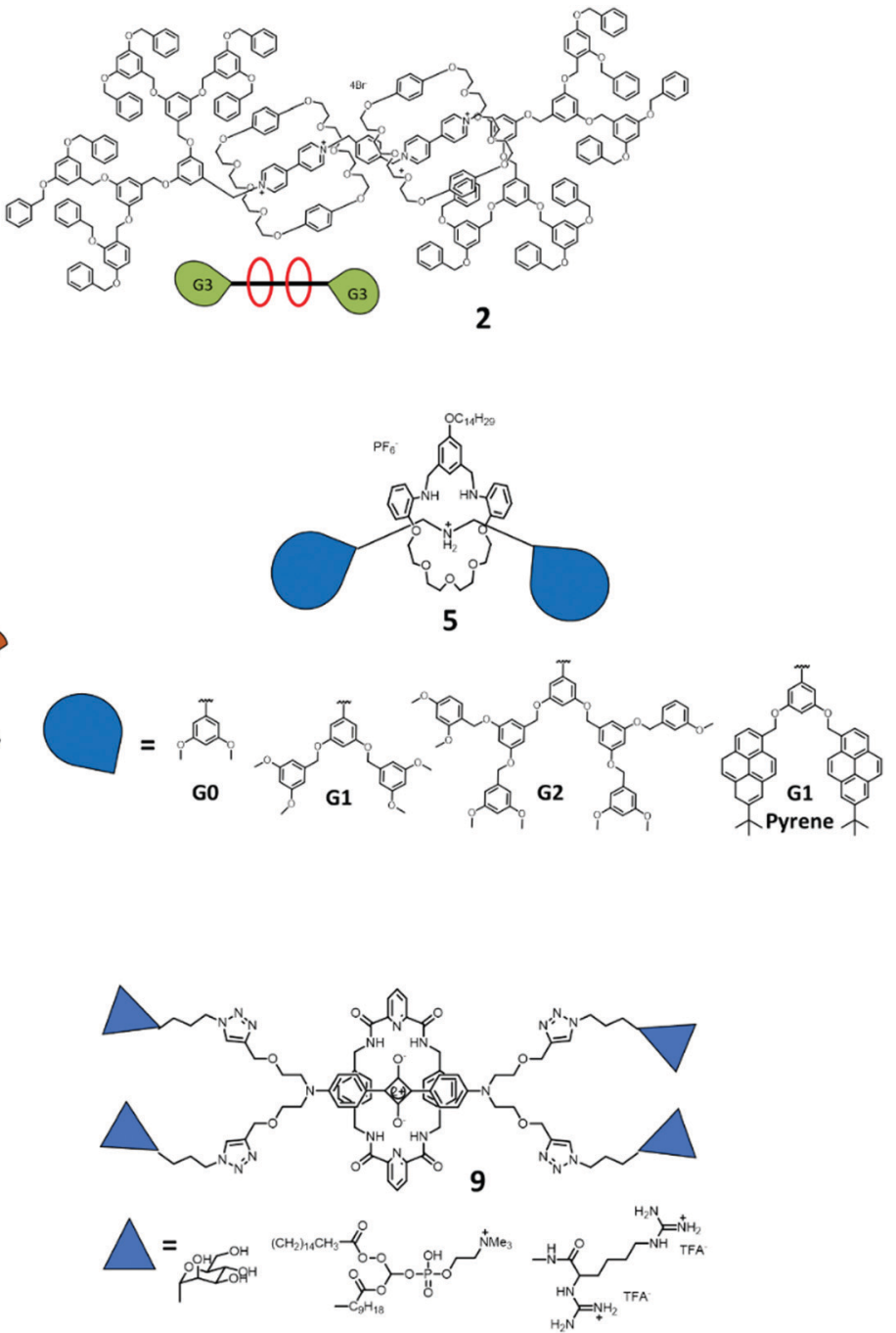
generation grafted into the rotaxanes, whereby on increasing the generation of the dendrons, it became more soluble in nonpolar solvents, such as toluene or benzene.

Liu and Yin et al. employed the dynamic covalent clipping synthesis $^{24}$ of G0 to G2 3,5-dimethoxy and a G1 tert-butyl pyrene-based dibenzylammonium (DBA) moiety with 2,6-pyridinedicarboxaldehyde and tetraethylene glycol bis(2-aminophenyl)ether. After reductive amination with a borane complex, a series of dendritic [2] rotaxanes $\mathbf{5}$ were produced. They also studied the photophysical properties of pyrene-based G1 stoppered [2]rotaxane.

Gao et al. prepared a bistable [2]rotaxane with a $\pi$-electrondeficient cyclobis(paraquat-p-phenylene) ring (CBPQT), tetrathiafulvalene (TTF) and 1,5-dioxynapthalene (DNP) as the pairs of $\pi$-electron-rich recognition sites, and azobenzene stoppering with a G2 Fréchet type benzyl ether stopper for a reproducible nanorecording thin film in $2005 .^{25}$ Later, Wang, Liu and $\mathrm{Li}$ et al. incorporated G3 dendrons into a tristable [2]rotaxane composed of CBPQT, TTF, DNP and azobenzene. ${ }^{26}$ Since part of the recognition moiety was not readily soluble, the use of G2 and G3 dendrons in these two examples was to increase the solubility in both polar and non-polar solvents.

Apart from the common Fréchet type benzyl ether dendrons, several different dendrons were also used as the stopper for rotaxane synthesis. Kato and Stoddart et al. used cyclohexylbiphenyl-derived mesogenic dendron stoppers for [2]rotaxane 6 synthesis and used compound 6 for a liquid crystal study (Fig. 2). ${ }^{27} \mathrm{Oh}$ and Lee et al. prepared [3] rotaxane 7 by a cucurbit[6]uril (CB[6]) promoted azide alkyne cycloaddition
(CB6AAC) dumbbell capture method with two G1 to G3 polyamindoamine dendrons. ${ }^{28}$ Kim et al. used similar dendrons for the donor-acceptor host guest interaction between methyl viologen and 2,6-dihydroxynaphthalene in $\mathrm{CB}[8]$ to form a pseudorotaxane host-guest complex 8. $^{29}$ Smith et al. reported a squaraine-based [2] rotaxane 9 stoppered with G1 to G3 N-Bocpolyamine and polyguanidinium dendrons or glucose (Fig. 2). ${ }^{30}$ The usage of these dendrons was to increase the water solubility and biocompatibility of the squaraine-based rotaxane for further biological imaging applications.

Among all literature reported examples of Type I-A rotaxane dendrimers, the chemical synthesis of Type I-A rotaxane dendrimers is relatively straight-forward and accessible in comparison to other types of rotaxane dendrimers. The attached dendrons usually act as bulky stoppers for the rotaxane core, thereby increasing the solubility and stability of the rotaxane component.

2.1.2 Type I-B rotaxane dendrimers. Type I-B rotaxane dendrimers are dendrons grafted onto macrocycles. Diederich et al. reported Fréchet type G1 to G2 dendritic cyclophanes for the formation of pseudo-rotaxanes with oligo(phenylacetylene) rods terminated with testosterone in water through hydrophobic interaction. ${ }^{31}$ Gibson et al. employed the G1 to G2 dendronfunctionalized DB24C8 for pseudo-rotaxane 10 formation with a tripodal DBA core (Fig. 3). ${ }^{32}$ They also studied the self-assembly of pseudo-rotaxanes in different solvents, including polar acetone and acetonitrile and relatively non-polar chloroform. The results indicated that larger dendrons on the rings increased the association constants $\left(K_{\mathrm{a}}\right)$ with tripodal DBA rods.
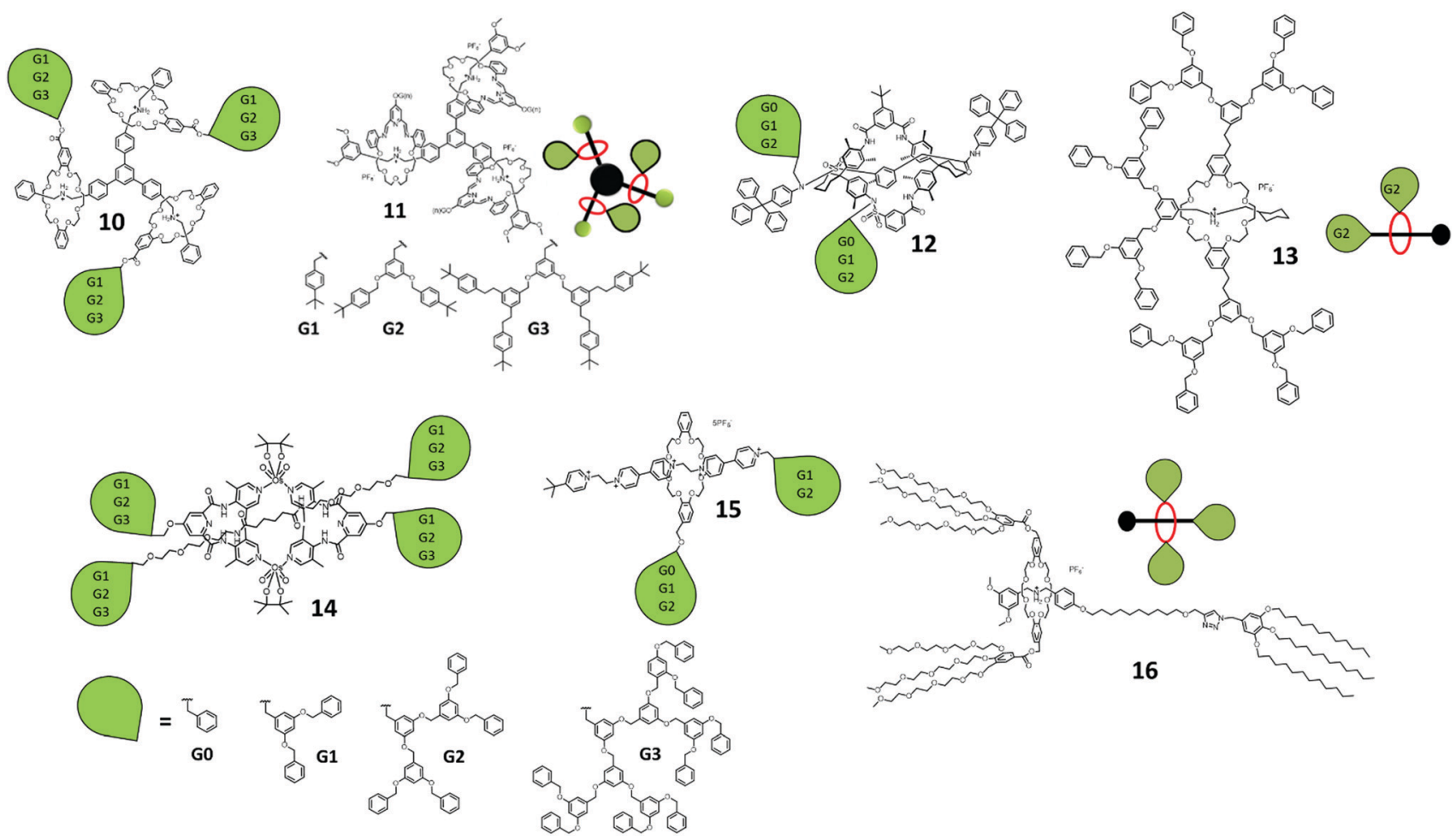

Fig. 3 Type I-B and I-C rotaxane dendrimers. 
Later, Stoddart et al. described the template-directed dynamic clipping synthesis of [4] rotaxane 11 through the imine clipping of G0 to G3 dendron-functionalized 2,6-pyridinedicarboxaldehyde and tetraethylene glycol bis(2-aminophenyl)ether with tripodal DBA followed by reduction (Fig. 3). ${ }^{33,34}$ With this strategy of dynamic covalent chemistry, they also explored the dynamic combinatorial libraries of these imine-based rotaxanes by mixing of different dendron grafted Type I-B rotaxane dendrimers. It is noteworthy that these examples of Type I-B tripodal rotaxane dendrimers can also be considered as Type II-A rotaxane dendrimers.

Newkome et al. introduced polycarboxylate dendrons on $\beta$-cyclodextrin as a host for pseudo-rotaxane formation with a dumbbell molecule containing two adamantane units as a guest. ${ }^{35}$ A more recent example was a dendron-functionalized pillar[5]arene (PA5) reported by Jia and Li et al. for pseudorotaxane formation. ${ }^{36}$ The G1 and G2 conjugated PA5 were selfassembled with tripodal pentanenitrile through hydrophobic interaction forming a pillar[5]arene-adiponitrile host-guest complex.

2.1.3 Type I-C rotaxane dendrimers. Type I-C rotaxane dendrimers are dendrons both grafted on the dumbbells and rings of the rotaxane. Vögtle et al. first introduced a Type I-C rotaxane dendrimer by the formation of tetralactam [2] rotaxane 12 followed by a chemoselective substitution of G1 to G3 dendrons with the sulfonamide groups in both the rings and rods, and studying the chiroptical phenomena of the chiral rotaxanes (Fig. 3). ${ }^{37}$

Stoddart et al. employed a slippage approach ${ }^{38}$ for the formation of [2]rotaxane 13 (Fig. 3). A G2 dendron-difunctionalized DB24C8 and a alkylammonium terminated with G2 dendrons and cyclohexyl were prepared for the formation of a [2] rotaxane. The G2 dendron-difunctionalized DB24C8 was thread through the cyclohexyl moiety after 90 days of refluxing, and deslipped slowly (4 days) in polar $d_{6}$-DMSO solvent at room temperature. Jeong et al. demonstrated a osmium-bridged metallocycle-based dynamic [2] rotaxane $\mathbf{1 4}$ with the attachment of G1 to G3 dendrons on both the macrocycles and threads (Fig. 3). ${ }^{39}$ The formation of rotaxane involved osmylation, coordination and hydrogen bonding in one-pot synthesis with pyridine-based ligands, an amide-containing thread and osmium tetroxide. Loeb et al. introduced also a Type I-C [2]rotaxane in the same article as the Type I-A [2]rotaxane $15,{ }^{23}$ involving the grafting of G0 to G2 dendrons on the rings and G1 to G2 on the dumbbells and studied the effect of the dendrons on the molecular shuttling kinetics (Fig. 3).

Recently, Qu et al. presented (Fig. 3) an acid-base responsive amphiphilic [2]rotaxane $\mathbf{1 6}$ and demonstrated a supramolecular morphology change in TEM upon pH switching. ${ }^{40}$ The [2]rotaxane was synthesized by oligo(ethylene glycol) di-functionalized DB24C8, where the dumbbell consisted of an alkyl chain stopper. The amphiphilic [2] rotaxane was self-assembled into micelles in water and turned into worm-like micelles upon the addition of base.

To this end, Type I rotaxane dendrimers possess the simplest chemical structures by introducing different types and generation of dendrons through either ring, thread or both.
Most of them were not switchable in nature, yet some of the examples showed potential applications for liquid crystal materials and micelle-like formation. In view of the above examples, the largest dendrons were generation three. The only challenge in Type I is the grafting of $G>3$ functional dendrons onto the rotaxane for more applications.

\subsection{Type II rotaxane dendrimers}

Type II rotaxane dendrimers are referring to the rotaxane connected to the outermost/peripheral branches of the dendrimer macromolecules/hypervalent core. The macromolecules here were reported as dendrimers, benzene-based components, porphyrinoids, metal complexes, [60]fullerene and many others.

2.2.1 Type II-A rotaxane dendrimers. Tripodal and tetrapodal $[n]$ rotaxanes with branched dumbbell structures can be classified as either Type II-A or Type III-A rotaxane dendrimers by definition if it is G1. We then discuss and classify all the G1 tripodal, tetrapodal and oligopodal rotaxane dendrimers as Type II-A rotaxane dendrimers.

Many of the earliest literature examples of Type II-A rotaxane dendrimers were pseudo-rotaxane in nature based on the hostguest interactions of different macrocycles with guest molecules. Based on the host-guest interaction between $\beta$-cyclodextrin and ferrocene, Kaifer et al. reported a series of ferrocenyl-conjugated poly(propylene imine) (PPI) core (G1-3) dendrimers terminated with up to $16 \beta$-cyclodextrin pseudo-rotaxane dendrimers. ${ }^{41}$ Later, the same group reported another series of cobaltoceniumfunctionalized PPI core (G1-4) dendrimers terminated with 32 $\beta$-cyclodextrin. ${ }^{42}$ The encapsulation of $\beta$-cyclodextrin significantly increased the water solubility of the dendrimers.

Another series of adamentyl-terminated PPI (G1-5) encapsulated with $\beta$-cyclodextrin were demonstrated by Meijer et al. They studied the $\mathrm{pH}$ effect of the host-guest interaction for the formation of a pseudo-rotaxane and used it as a nanoreactor for the preparation of gold and platinum nanoparticles. ${ }^{43}$ The stable interaction between protonated diaminobutane and cucurbituril ( $\mathrm{CB}[6])$ was also applied into the G1-5 PPI by Kim et al. Up to $64 \mathrm{CB}[6]$ were encapsulated on the surface of G5 PPI. ${ }^{44}$ They also studied the morphology through atomic force microscopy (AFM) and the dethreading effect of $\mathrm{CB}[6]$ upon the addition of base.

The usage of the recognition between DB24C8, DBA and $N$-methyltriazolium (MTA) units to form tripodal and tetrapodal [4]rotaxanes was reported by Qu et al. and Osakada et al. Qu used the tripodal [4]rotaxane 17 for fluorescence modulation by photoinduced electron transfer (PET) upon the shuttling of the di-ferrocene-linked DB24C8 to the MTA near the 4-morpholinonaphthalimide unit (Fig. 4). ${ }^{45}$ Another tetrapodal [5]rotaxane 18 was reported by Qu using the same di-ferrocene-linked DB24C8 with the simple 3,5-dimethoxyl stopper while the core was the $\mathrm{Zn(II)}$ porphyrin (Fig. 4). ${ }^{46}$ Osakada reported the synthesis of ferrocene terminated tripodal [4] rotaxane 19 by CuAAC, showing the rotaxane crystal structure (Fig. 4). ${ }^{47}$

The approach of using active metal template (AT) synthesis of tripodal and tetrapodal $[n]$ rotaxanes was developed by Goldup et al. using the AT-copper catalyzed azide alkyne 


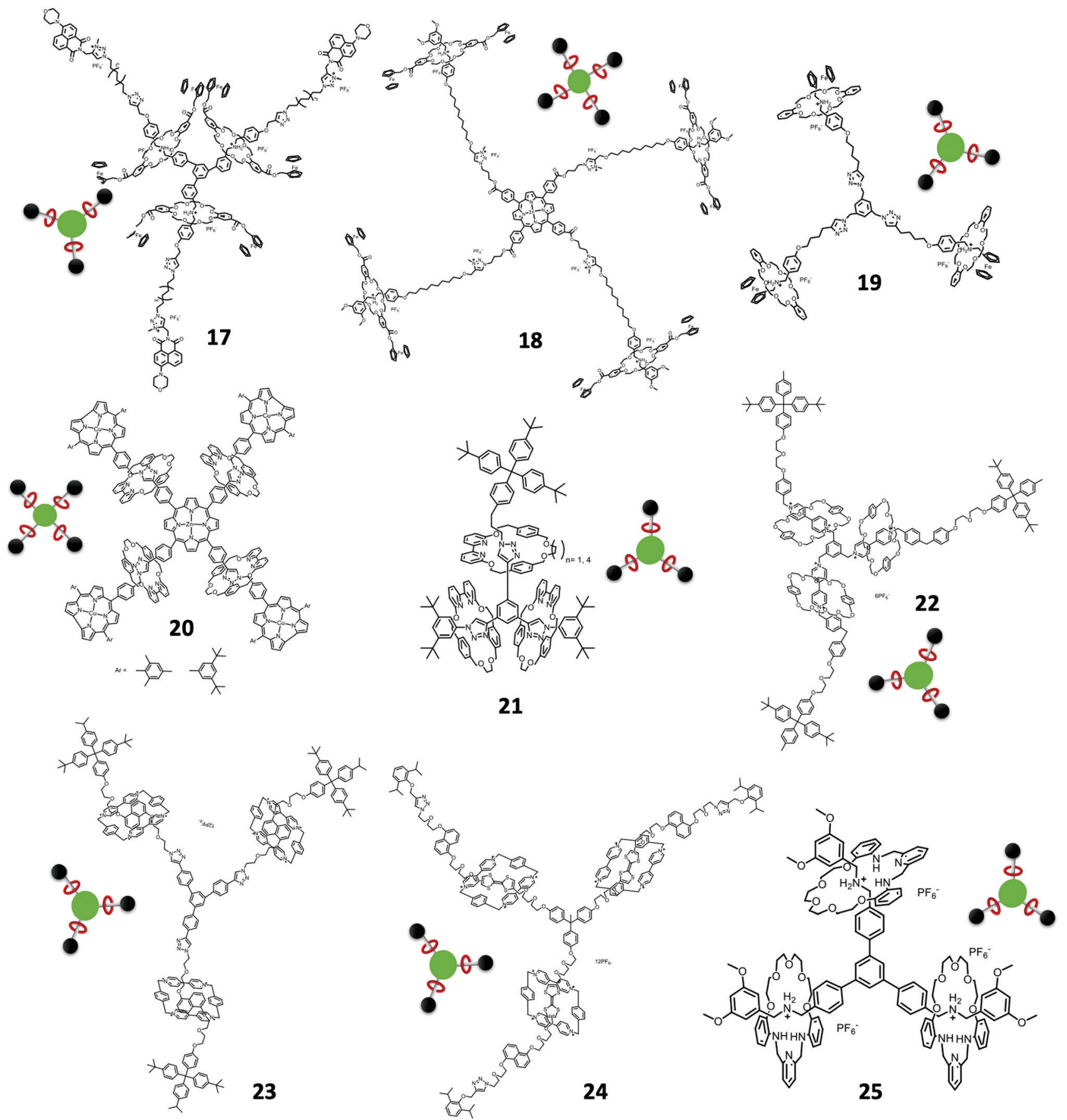

Fig. 4 Tripodal and tetrapodal Type II-A rotaxane dendrimers.

cycloaddition (AT-CuAAC) reaction. This approach required the usage of 2,2'-bipyridine (bpy) based macrocycles. A tetrapodal [5]rotaxane 20 with the $\mathrm{Zn}$ (II) porphyrin core and $\mathrm{Cu}$ (II) corrole stopper encircled by bpy macrocycles was synthesized through AT-CuAAC (Fig. 4). ${ }^{48}$ Dimerization of the $\mathrm{Zn}$ (II) porphyrin core [5]rotaxane with ditopic ligand 1,4-diazabicyclo[2.2.2]octane (DABCO) and the photophysical properties were also studied in the paper. Using the same approach, Goldup's group also investigated the stepwise and protecting group free synthesis of tripodal [4]rotaxane 21 (Fig. 4). ${ }^{49}$ The synthesis involved the formation of one mechanical bond in each step and finally gave the tripodal [4] rotaxanes with two different size of bpy-based macrocycles.

Stoddart et al. employed the concept of a $\pi$-donor and $\pi$-acceptor templating approach for tripodal [4]rotaxane $\mathbf{2 2}$ synthesis (Fig. 4). ${ }^{50}$ The first synthesis of this dendritic rotaxane was by the recognition of $\mathrm{BIPY}^{2+}$ and BPP34C10 using the slippage of BPP34C10 to the tripodal thread. ${ }^{11}$ They later 
reported the recognition of CBPQT and DNP for [4]rotaxane formation. CBPQT $\supset$ DNP azide pseudorotaxane was clicked with the tris(propargyl ether) to give the [4]rotaxane. Similarly, the recognition of CBPQT $\supset$ TTF was also used for the synthesis of [4]rotaxanes $23^{51}$ and 24 (Fig. 4). ${ }^{52}$ Stoddart et al. also employed the dynamic clipping of 2,6-pyridinedicarboxaldehyde with tetra(ethylene glycol) bis(2-aminophenyl)ether followed by imine reduction by $\mathrm{BH}_{3}$.THF (borane tetrahydrofuran complex) in the synthesis of tripodal [4]rotaxane 25 (Fig. 4). ${ }^{53}$

A cucurbit[6] uril-promoted click reaction was employed by Steinke and Tuncel et al. for the synthesis of tripodal mesitylene core and tetrapodal Zn(II) porphyrin core [5]rotaxane 26 (Fig. 5). ${ }^{54}$ The same group recently investigated the $\mathrm{CB}[6]$-porphyrin based [5]rotaxane 27 as a photosensitizer for photodynamic therapy (PDT) (Fig. 5). ${ }^{55}$ The $\mathrm{CB}[6]$-based rotaxane gave excellent water solubility of the porphyrin, and exhibited antibacterial properties towards $E$. coli, B. subtilis and the MCF7 cell line upon white light radiation.

Yang et al. also prepared a tetrapodal $\mathrm{Zn(II)} \mathrm{porphyrin} \mathrm{core}$ [5]rotaxane through the platinum-acetylide formation with platinum iodine terminal pillar[5] arene [2]rotaxane with tetraacetylene $\mathrm{Zn}$ (II) porphyrin and studied their aggregation behaviors. ${ }^{56}$ Tokunaga et al. used another approach of forming the [4] rotaxane $\mathbf{2 8}$ with boroxine condensation through boronic acids (Fig. 5). ${ }^{57}$ The synthesis involved the self-assembly of DB24C8 and boronic acid terminated-DBA followed by boroxine condensation giving the tripodal boroxine [4]rotaxane. Kihara and Takata et al. synthesized a tripodal [4]rotaxane 29 via the hydrosilylation of the alkyne in the terminal of the thread catalyzed by $\mathrm{RuHCl}(\mathrm{CO})\left(\mathrm{PPh}_{3}\right)_{3}$ under ambient conditions (Fig. 5). ${ }^{58}$

Winpenny et al. designed an organic-inorganic hybrid approach for the synthesis of polyrotaxanes. $\mathrm{A} \mathrm{Cr}_{7} \mathrm{Ni}$ heterometallic ring was able to form the rotaxane with an alkyl ammonium thread, while the thread was terminated with pyridine. This organic-inorganic hybrid rotaxane can then be coordinated with $\left[\mathrm{Fe}_{2} \mathrm{Co}(\mathrm{O})\left(\mathrm{O}_{2} \mathrm{CtBu}\right)_{6}\left(\mathrm{H}_{2} \mathrm{O}\right)_{3}\right]$ to form the [4] rotaxane and [7]rotaxane organic-inorganic hybrid dendrimers 30 though a convergent approach. The organic-inorganic hybrid rotaxane dendrimers were further confirmed by X-ray crystallography (Fig. 5). ${ }^{59}$

Other higher order Type II-A rotaxane dendrimers were reported by Qu et al. as a hexa-branched [7]rotaxane 31 synthesized from alkyne-containing [3]rotaxane via a cobalt-catalyzed $[2+2+2]$ alkyne cyclotrimerization (Fig. 5) ${ }^{60}$ The key intermediate of hexa-branched [7]rotaxane was a [3]rotaxane containing two identical stopper recognition sites and macrocycles where the middle part linked by an alkyne (1,2-diphenylethyne) group. [3] rotaxane was further reacted with $10 \mathrm{~mol} \%$ of $\mathrm{Co}_{2}(\mathrm{CO})_{8}$ in

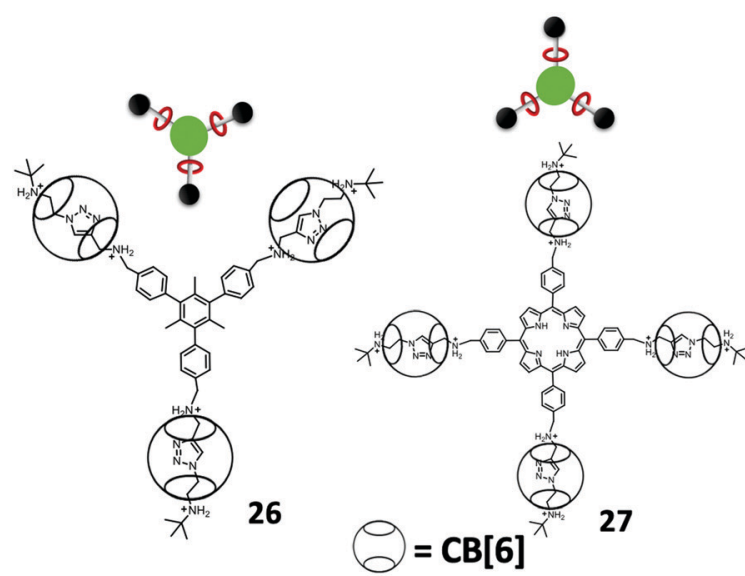

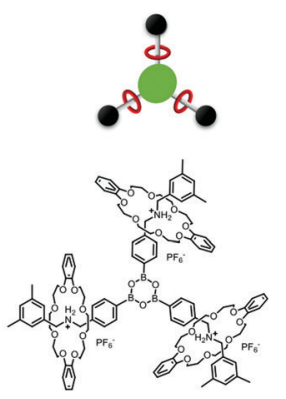

28

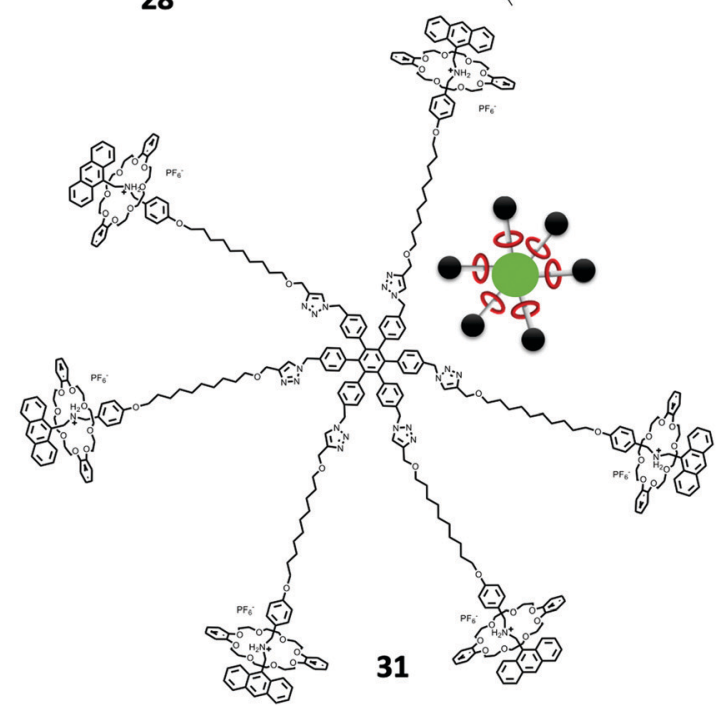

Fig. 5 Tripodal and hexapodal Type II-A rotaxane dendrimers. (Copyright: Nature Publishing Group for 30.) 
dioxane to give the desired hexa-branched [7]rotaxane in $20 \%$ yield by $[2+2+2]$ alkyne cyclotrimerization. The core of the hexabranched [7]rotaxane was hexaphenyl benzene, which prevents coplanar conformations.

Yang et al. employed the coordination-driven self-assembly strategy for synthesizing a supramolecular metalacyclic core with rotaxane units on the periphery (Fig. 6A). The supramolecular metalacyclic cores were based on Pt-acetylides building blocks. In another part, a pillar[5]arene and neutral alkyl chain based [2] rotaxane terminated with a Pt-acetylide unit was synthesized. This PA5-[2]rotaxane was coupled with two different (mono/di)alkynyl bis-pyridine precursors to form the building blocks. By varying the pyridine ligands used in the selfassembly, a series of Type II-A rotaxane dendrimers were obtained, including square/hexagonal cores, terminated with different numbers of rotaxane units. ${ }^{61}$ The disassembly/ reassembly process of the supramolecular metallacyclic cores can be performed with silver or bromide ions.

In view of all the Type II-A rotaxane dendrimers described above, they all have a G1 core, while core $G>2$ gave pseudorotaxane host-guest complexes. The only example of a core $G>2$ Type II-A rotaxane dendrimer 32 (Fig. 6B) was achieved by Yang and co-workers. ${ }^{62}$ Their approach was begun with the core Pt-acetylide based organometallic dendrimer core (G1-4) synthesis through a divergent approach whereas the dendrimers were terminated by a triisopropylsilane (TIPS) protecting group. The synthesized G1-4 Pt-based dendrimers were coupled with the PA5-based [2] rotaxane via a $\mathrm{Cu}(\mathrm{I})$ catalyzed coupling reaction allowing the formation of 24 new mechanical bonds in a one pot reaction for the G4 [25]rotaxane dendrimer. All the synthesized organometallic based Type II-A rotaxane dendrimers were characterized thoroughly by NMR, MS, GPC, DLS and AFM. These results showed that an increase of generation will increase the sizes of the observed particles on a mica surface and were consistent with the TEM images.

2.2.2 Type II-B rotaxane dendrimers. Type II-B rotaxane dendrimers are dendrimers with macrocycles at the dendrimer's surface, interlocking with rod components. Most of the reported Type II rotaxane dendrimers are within Type II-B rotaxane dendrimers. By varying the core molecules, bis, tris, tetrakis and multiple Type II-B rotaxane dendrimers can be synthesized.

Stoddart et al. first designed and synthesized a bis[2]rotaxane 33 in 1994, using a template-directed synthesis
(Fig. 7). ${ }^{63,64}$ Two identical threads containing electron rich 1,5-dioxynapthlene were obtained and the last step was the formation of a bis-CBPQT with two identical threads forming the bis[2]rotaxane. Stoddart et al. later used the dynamic imine clipping method to synthesize bis[2] rotaxane 34 (Fig. 7). ${ }^{65}$ The usage of the imine clipping method in rotaxane dendrimer synthesis involved two 2,6-pyridinedicarboxaldehyde units (tetraaldehyde) with the normal bis(3,5-dimethoxybenzyl)ammonium hexafluorophosphate and tetraethyleneglycol bis(2-aminophenyl)ether. After the dynamic rotaxane was formed, $\mathrm{BH}_{3}$. THF solution was introduced to reduce the imine to form the rotaxane in 96\% yield. Stoddart et al. also utilized the common DB24C8 and DBA interaction for the synthesis of dendritic-stopper bis[2]rotaxane 35 (Fig. 7). ${ }^{66}$ An ester linked bis-DB24C8 and the bis[2]rotaxane bearing benzylic triphenylphosphonium ion stoppers were synthesized. Finally, dendritic ether stoppers were exchanged with the triphenylphosphonium ions via a Witting reaction to form the dendritic-stopper Type II-B rotaxane dendrimers.

Loeb et al. used another interaction between DB24C8 and 1,2-bis(4,4-dipyridinium)ethane axle for the formation of [3]rotaxane 36 (Fig. 7). In the synthesis of Type II-B rotaxane dendrimers, they first synthesized three bis(crown) ethers, including different spacers with different arene substitution patterns 1,2-, 1,3-, 1,4- and biphenyl bisDB24C8. ${ }^{67}$ Their findings suggested that the steric hindrance affected the formation of the Type II-B rotaxane dendrimers, where the 1,4-position gave the best yield of rotaxane.

$\mathrm{Qu}$ et al. reported an overcrowded alkene switchable [3]rotaxane 37 (Fig. 7). ${ }^{68}$ The synthesis first involved a bis-crown ether linked with trans-alkene. Two dihydroxyl-functionalized DBA threads were recognized by the crown ether and stoppered by tri- $n$-butylphosphine-catalyzed esterification. The photoinduced trans-to-cis isomerization of the central double bond of the rotaxane was characterized by NMR, UV-Vis and fluorescence spectra. The isomerization switching clam-like motion was reversible by external light and thermal stimuli.

Other than the common method for the formation of Type II-B rotaxane dendrimers, there are several other reported methods for the synthesis. Asakawa and co-workers used a bis-palladium(II)-salophen macrocycle for the formation of bis[2] rotaxane $\mathbf{3 8}$ by a threading-followed-by-shrinking approach (Fig. 7). ${ }^{69}$ Crowley et al. synthesized an iron(II)-based coordination
A

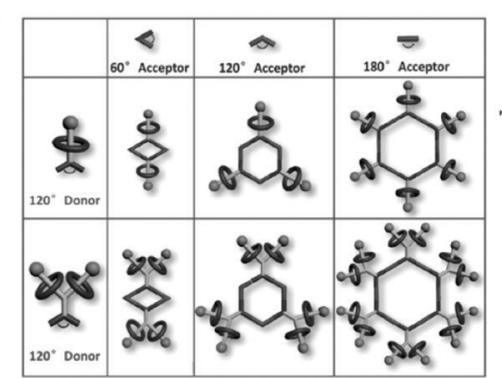

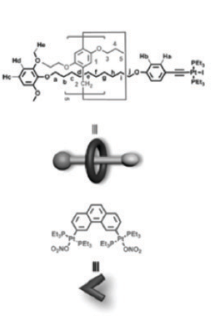

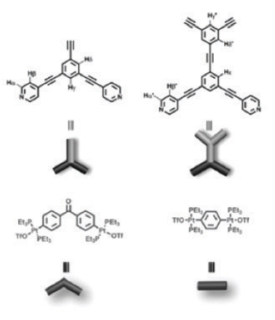

B

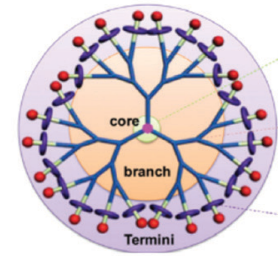

32

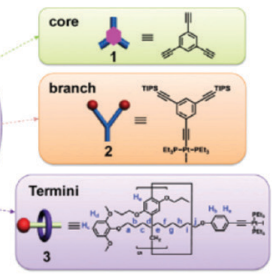

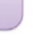

Fig. 6 Yang's Type II-A rotaxane dendrimers. (Copyright: Wiley for A, Royal Society of Chemistry for B.) 


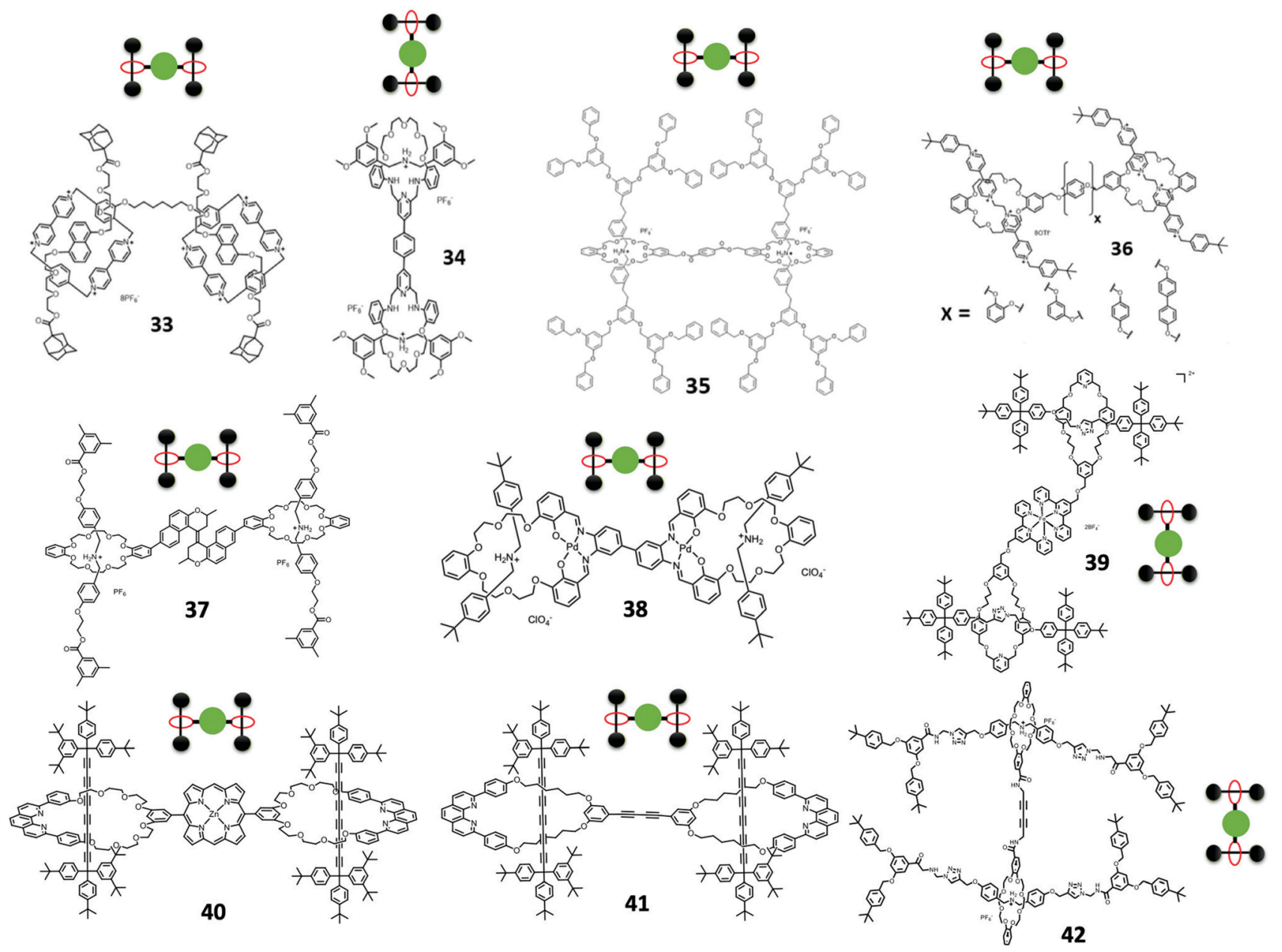

Fig. 7 Bis-macrocycle Type II-B rotaxane dendrimers.

complex bis[2]rotaxane 39 (Fig. 7). ${ }^{70}$ The first step involved an active-metal template synthesis of a macrocycle-functionalized [2]rotaxane and the methyl alcohol functional group of the macrocycle was further changed to terpyridine. By mixing $\left[\mathrm{Fe}\left(\mathrm{H}_{2} \mathrm{O}\right)_{6}\right]\left(\mathrm{BF}_{4}\right)_{2}$ with the terpyridine functionalized [2] rotaxane, diamagnetic low-spin iron(II) Type II-B bis[2]rotaxane was synthesized.

Anderson et al. used an aldehyde functionalized phenanthroline-based macrocycle with active metal template Cadiot-Chodkiewicz coupling to synthesize a polyyne [2]rotaxane accompanied by an aldehyde functional group. The aldehyde functionalized rotaxane was then condensed to porphyrin in the presence of dipyrromethane, trifluoroacetic acid (TFA) and 2,3-dichloro-5,6-dicyano-1,4-benzoquinone (DDQ) to give porphyrinbased [3] rotaxane 40 in $62 \%$ yield (Fig. 7). ${ }^{71}$ Tykwinski et al. lately also reported a [3]rotaxane $\mathbf{4 1}$ synthesis using the similar CadiotChodkiewicz coupling for the polyyne [2]rotaxane followed by Glaser-Hay homocoupling on two macrocycles (Fig. 7). ${ }^{72}$ Leung et al. employed this Glaser-Hay homocoupling of two propargyl functionalized-DB24C8 [2] rotaxanes to make a [3]rotaxane 42 Type II-B rotaxane dendrimer in $60 \%$ yield (Fig. 7 ), a prototype in making Type III-B rotaxane dendrimers. ${ }^{73}$
Among the Type II-B rotaxane dendrimers, bis-macrocycle Type II-B rotaxane dendrimers were relatively easy to synthesize because of the less steric hindrance to the core of the rotaxane dendrimers.

Loeb et al. reported the preparation of tris-macrocycles [4] rotaxane 43 Type II-B rotaxanes based their on their finding in bis-macrocycle Type II-B rotaxane dendrimers (Fig. 8). ${ }^{67}$ Also, tris-DB24C8 used the same recognition between DB24C8 and 1,2-bis(4,4-dipyridinium)ethane for the synthesis of [4]rotaxane in $35 \%$ yield.

Yang and Stang et al. prepared a series of tris(DB24C8) hexagons via coordination-driven self-assembly (Fig. 9A-C) ${ }^{74,75}$ The self-assembly involved a $120^{\circ}$ crown ether-containing diplatinum(II) acceptor with a $120^{\circ}$ dipyridyl donor for the formation of hexagon platinum complexes. They studied the formation of a pseudorotaxane of the hexagons with DBA ions, where the formation of the tris-pseudorotaxane can be started from the formation of pseudo-rotaxane with DBA ions and $120^{\circ}$ crown ether-containing diplatinum(II) acceptors and then coordination-driven self-assembly or the formation of hexagons followed by pseudo-rotaxane formation. By varying the ligands of the $120^{\circ}$ dipyridyl donors with G0 to G3 dendrons or the G1 

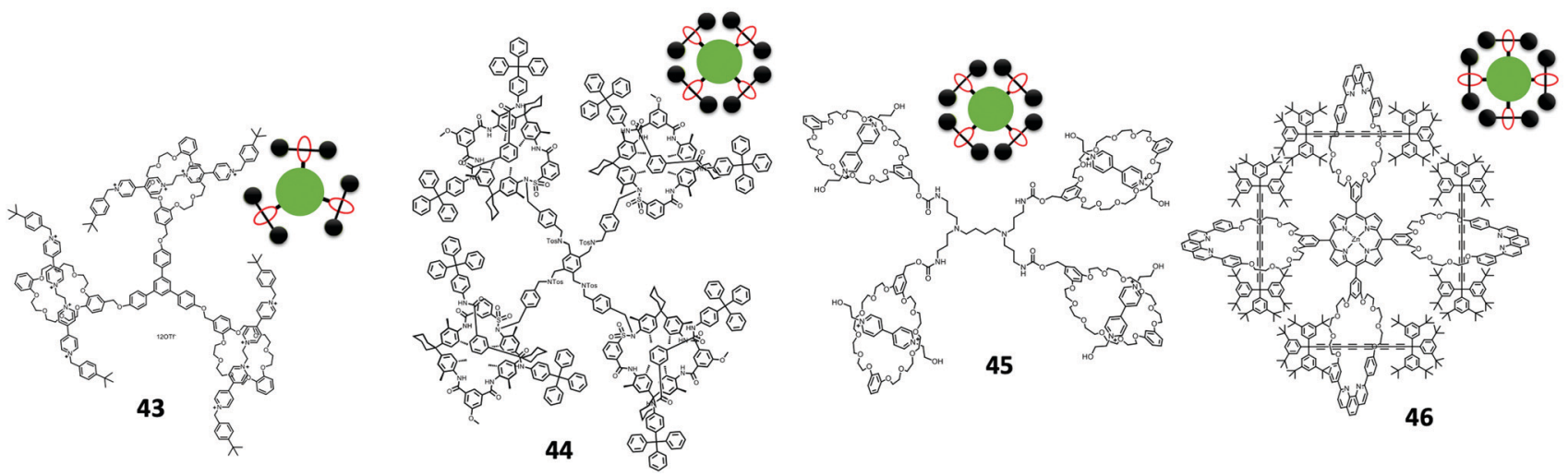

Fig. 8 Tris- and tetrakis-macrocycle Type II-B rotaxane dendrimers.

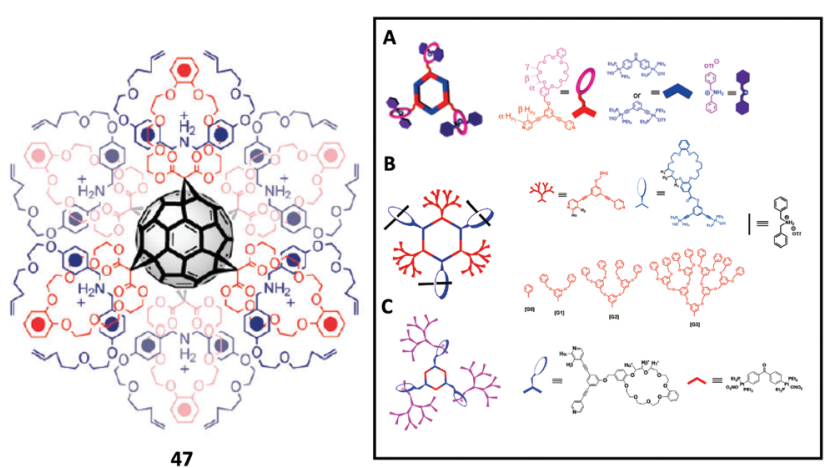

Fig. 9 Multiple-macrocycle and hexagon-based Type II-B rotaxane dendrimers. (Copyright: Royal Society of Chemistry for $\mathbf{4 7}$ and Wiley for A-C.)

to G3 dendritic DBA cations, ${ }^{76}$ they can perform the same coordination-driven self-assembly for the Type II-B pseudorotaxane dendrimers.

The first tetrakis-macrocycle Type II-B rotaxane dendrimer was reported by Vögtle et al. in $1999 .{ }^{77}$ Their synthesis began with the formation of [2]rotaxane with a functionalized monosulfonamide-based macrocycle, and this functionalized [2]rotaxane can form a tetrakis Type II-B rotaxane dendrimer by reacting it with a tetrabromide core molecules to form tetrakis [5]rotaxane 44 (Fig. 8).

Gibson et al. in 2003 used another approach to attempt synthesis of tetrakis pseudo-rotaxane 45 (Fig. 8).$^{78}$ They used a first generation poly(propyleneimine) (PPI) dendrimer as the core and changed the four amine groups into isocyanate and further reacted with a functionalized bis( $m$-phenylene)-32crown-10 to form tetrakis-macrocycles. The formation of a pseudo-rotaxane with $4,4^{\prime}$-bipyridinium derivatives and tetrakismacrocycles was also studied.

Tetrakis functionalized porphyrin [5]rotaxane 46 was synthesized by Anderson et al. with the same approach as the bismacrocycle Type II-B rotaxane dendrimers described above (Fig. 8). ${ }^{71}$ Since formation of porphyrin involved the condensation between benzaldehyde and pyrrole, this study showed that if the benzaldehyde directly connected to the [2] rotaxane, the tetrakis-functionalized porphyrin cannot be formed due to the steric bulk of the four [2] rotaxane substituents. However, if it had a $p$-phenylene spacer at the benzaldehyde functionalized [2]rotaxane, the porphyrin [5]rotaxane $\mathbf{3 1}$ can be successfully synthesized in $10 \%$ yield.

From the literature, there was only one example of a core with more than four macrocycles which was reported by Stoddart et al. In their report, they synthesized a hexakisadducts of [60]fullerene $\mathbf{4 7}$ bearing malonato-benzo[25]crown8 rings (Fig. 9).$^{79}$ After obtaining the hexakis-functionalized [60]fullerene, DBA derivative was used for the formation of [7]pseudorotaxanes and the threading and dethreading of DBA to the crown ether-functionalized [60]fullerene was slow on the ${ }^{1} \mathrm{H}$ NMR timescale.

2.2.3 Type II-C rotaxane dendrimers. A Type II-C rotaxane dendrimer was first defined by Stoddart et al. in 2016 as a rotaxane dendrimer where both the rings and their threaded axles are connected to the dendrimer's periphery. ${ }^{20}$ Since the connection of both macrocycle and thread can be one component in nature, from this definition, we further interpret and include the definition as the connection of multiple [1] rotaxane moieties into a core molecule.

Crowley et al. synthesized a [2]rotaxane $\mathbf{4 8}$ with triazole on the thread and a pyridine-based macrocycle (Fig. 10). ${ }^{70}$ This rotaxane can be dimerized with silver trifluoromethanesulfonate (AgOTf) to form a [1] rotaxane like structure, where both triazole (thread) and pyridine (macrocycle) attached to the Ag(I) core. As observed from the X-ray crystal structure, the size of the ring was not suitable for both $\mathrm{Ag}$ and pyridine, and it formed a dimer in which a $\mathrm{Ag}(\mathrm{I})$ ion bridged between the rotaxane. This example showed that both the rings and threads were directly attached to the $\mathrm{Ag}(\mathrm{I})$ (core) as one type of Type II-C rotaxane dendrimer.

Apart from this example, Qu et al. reported the tris[1]rotaxane 49 and tetrakis[1] rotaxane $\mathbf{5 0}$ star-like structures (Fig. 10) ${ }^{80} \mathrm{~A}$ tri- and tetra-azide functionalized core and a di-substituted ferrocene component composed of both DB24C8 and DBA were synthesized. The di-substituted ferrocene was then self-assembled itself and clicked with a tri-/tetra azide core to form tris[1]rotaxane and tetrakis[1]rotaxane, respectively. By the methylation of triazole to MTA, acid-base switching was achieved 


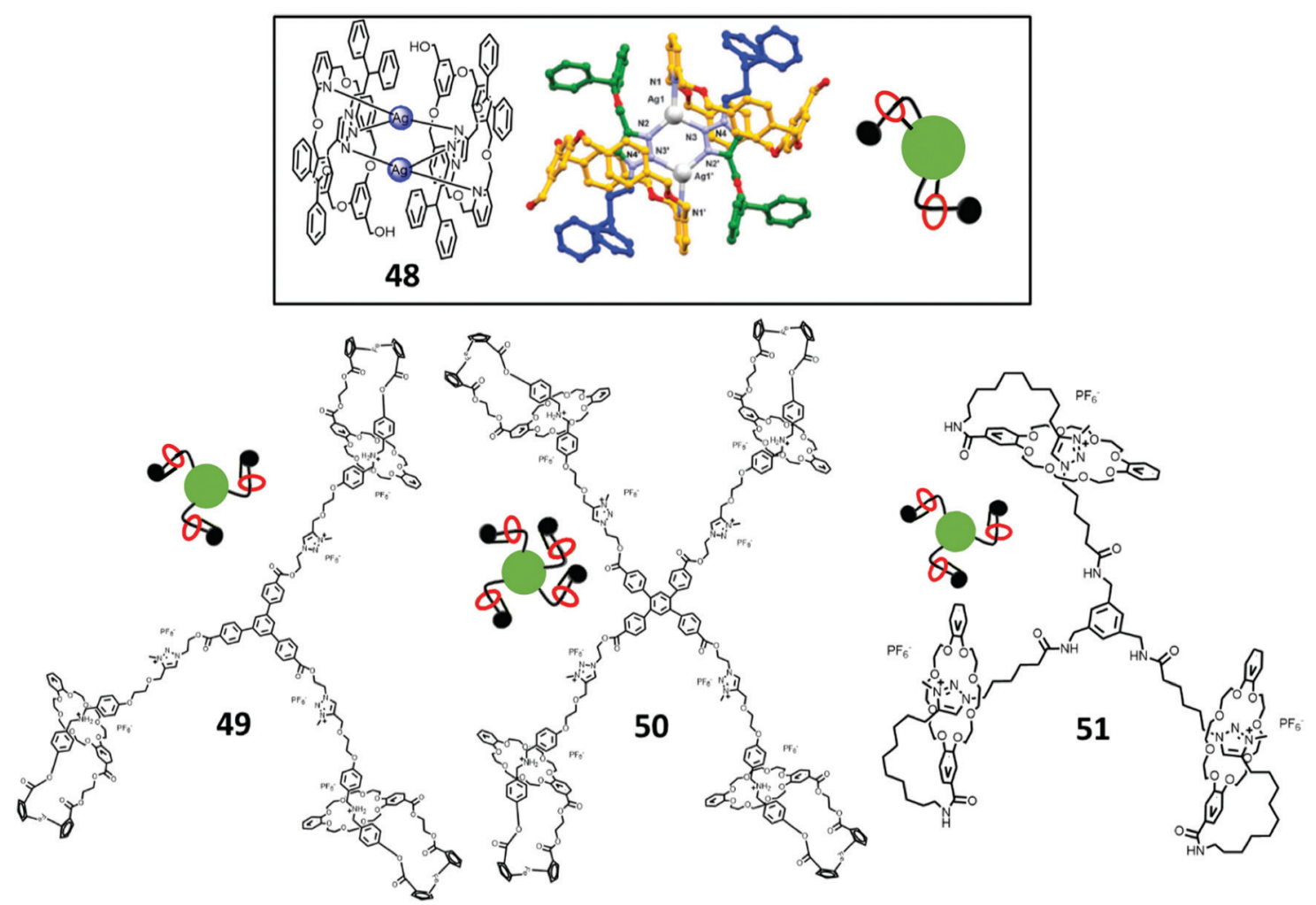

Fig. 10 Type II-C rotaxane dendrimers. (Copyright: Royal Society of Chemistry for $\mathbf{4 8}$ crystal structure.)

and mimicked the extension and contraction motions in the molecules.

Coutrot et al. in 2015 synthesized a tris[1] rotaxane 51 using a 'macrocycle transporter' approach (Fig. 10). ${ }^{81}$ A thread containing DBA and a $N$-hydroxysuccinimide (NHS) moiety was synthesized. The [1] rotaxane was then synthesized as a transporter by functionalizing the NHS hydroxy group with azide and self-clicked to the alkyne-linked DB24C8, and the triazole was further methylated to MTA afterwards. To achieved the transporter [1] rotaxane, the macrocycle was shuttled to MTA by $N$-carbamoylation of DBA by $\mathrm{Boc}_{2} \mathrm{O}$. Finally, by reacting a tripodal phenyl methylamine molecule with the transporter [1]rotaxane, tris[1] rotaxane was synthesized.

To this end, the molecular complexity of Type II rotaxane dendrimers increased, and various shuttling behaviors were achieved in this category. Some of the examples of Type II rotaxane dendrimers such as $\mathbf{2 6}$ and $\mathbf{2 7}$ were capable of gaining water solubilities. Although not many of them showed applications, a number of sophisticated synthetic reactions including AT-CuAAC, cucurbit[6]uril-promoted click reaction, [2+2+2] alkyne cyclotrimerization, Cadiot-Chodkiewicz coupling and Glaser-Hay homocoupling were applied in the synthesis of Type II rotaxane dendrimers thereby giving an advance for the synthesis of more complicated rotaxane dendrimer structures. There is still a challenging synthetic task within this category, which is the synthesis of a $G>2$ dendritic core for the Type II rotaxane dendrimers. So far, there is only one example from Yang's group in Type II-A, and this leaves big room for synthetic chemist to achieve other core $G>2$ Type II (A, B, C) rotaxane dendrimers.

\subsection{Type III rotaxane dendrimers}

Type III rotaxane dendrimers are referring to dendritic polyrotaxane architectures, where the mechanical bonds are branched like a dendrimer molecule. In the rotaxane dendrimer family, Type III rotaxane dendrimers have the fewest examples in the literature especially the higher generation ones due to their structure complexity.

2.3.1 Type III-A rotaxane dendrimers. In Type III-A rotaxane dendrimers, multiple macrocycles are encircling in between the dendrimer branches, whereas the mechanical bonds are in between the dendrimer scaffold's branching point.

Among all examples shown above (including Type II-B rotaxane dendrimers), Type III-A rotaxane dendrimers with $G>2$ have not been reported. The very first G2 (pseudo)rotaxane dendrimer 52 was reported by Kim et al. in 2007 through a divergent approach (Fig. 11). ${ }^{82}$ The starting step was the formation of a tripodal trans-1,2-bis(1-methyl-4-pyridinio)ethylene, where this moiety forms a stable 1:1 complex with $\mathrm{CB}[8]$ in $\mathrm{D}_{2} \mathrm{O}$. In another (pseudo)rotaxane, it is composed of two branched diaminobutane units for the formation of a stable 1 : 1 complex with $\mathrm{CB}[6]$ and one free dihydroxynaphthalene. Since the cavity of $\mathrm{CB}[8]$ is relatively large, it can encapsulate more guests even after forming one host-guest complex. This electron rich dihydroxynaphthalene pseudorotaxane finally bound with the tripodal trans-1,2-bis(1-methyl-4-pyridinio)ethylene $\mathrm{CB}[8]$ 


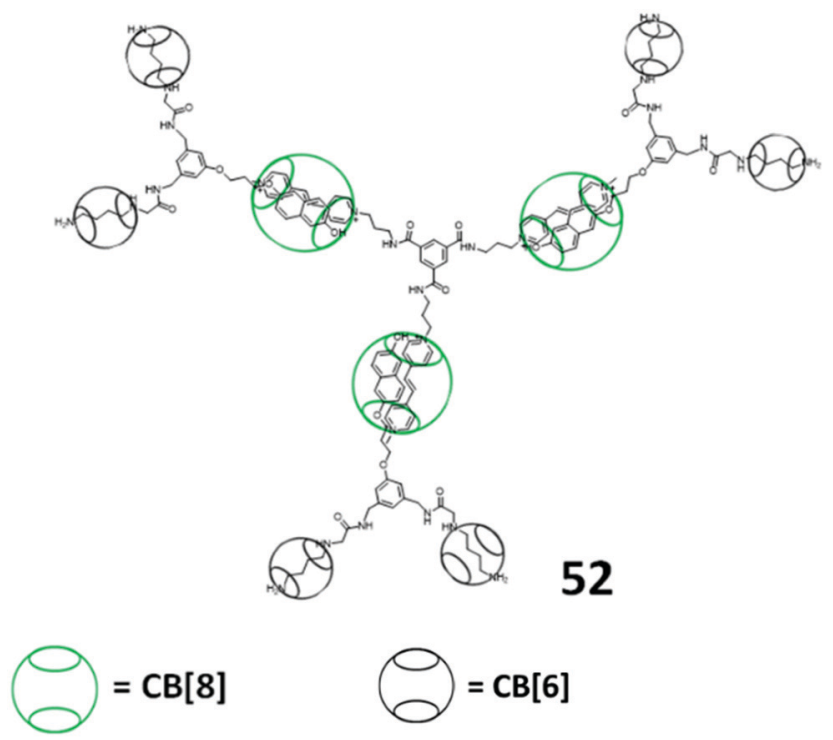

Fig. 11 Kim's Type III-A pseudo-rotaxane dendrimer.

host-guest complex to form a stable [10]pseudorotaxane ternary complex in $\mathrm{D}_{2} \mathrm{O}$.

In 2015, an example of a $G>2$ Type III-A rotaxane dendrimer was first reported by Yang's group where they synthesized up to G4 [46]rotaxane dendrimers containing 50 mechanical bonds in one macromolecule (Fig. 12A). ${ }^{83}$ Their synthesis involved a divergent approach for synthesizing high generation Type III-A rotaxane dendrimers. The basic backbone of these Type III-A rotaxane dendrimers was based on G0 PA5 [2]rotaxane. This G0 PA5-based [2] rotaxane was terminated with one stopper with a platinum iodide acetylide complex and the opposite stopper was dendritic triisopropylsilyl (TIPS)protected 3,5-diethynylbenzene units. Three [2]rotaxanes were reacted with 1,3,5-triethynylbenzene through $\mathrm{Cu}$-catalyzed hetero-coupling to form G1 with new platinum acetylide bonds and terminated with six TIPS stoppers. The TIPS stoppers were deprotected by $\mathrm{NBu}_{4} \mathrm{~F}$ to generate free branching point terminal alkynes. By repeating the same step of reacting $\mathrm{G} 0$ with deprotected G1, a G2 rotaxane dendrimer was formed. The formation of G3 and G4 Type III-A rotaxane dendrimers also employed the same strategy of deprotection and heterocoupling. All Type III-A rotaxane dendrimers were characterized thoroughly by NMR, MALDI-TOF MS and showed monodispersed morphology in AFM analysis.

Later in 2018, the same group prepared other similar up to G3 [22] rotaxane dendrimers with dual (solvent and anion) stimuli-responsive properties and demonstrated a reversible dimension modulation (Fig. 12B). ${ }^{84}$ The design of the new stimuli-responsive pillar[5]arene-based Type III-A rotaxane dendrimers involved introducing a urea moiety into the thread to serve as a stimuli-responsive site, and the other parts and synthetic strategy were similar to the one reported in 2015. The PA5 in the rotaxanes sat on the urea site. Since the new Type III-A rotaxane dendrimers contain urea groups, the addition of dimethylsulfoxide (DMSO) (solvent) or tetrabutylammonium acetate (TBAA) (anion) formed complementary binding with urea and PA5 was moved towards the core alkyl chain. Such shuttling processes were confirmed by NMR and could be reversed by the addition of $\mathrm{NaPF}_{6}$ to get a NaOAc precipitate. The author further showed that the movement of macrocycles on the branches may affect the folding behaviors of the dendrimers thus leading to the size shrinkage observed from DOSY and AFM.

In addition, Yang's group introduced aggregation-induced emission (AIE) behavior into the same backbones of ureapillar[5]arene-based Type III-A rotaxane dendrimers (Fig. 12C). ${ }^{85}$ The core of the macromolecules was changed from 1,3,5-triethynylbenzene to diethynyl 9,10-distyrylanthrance to give AIE active G1 to G3 rotaxane dendrimers. The results indicated that different generations gave different AIE colors, and an increase of generation can give more obvious AIE activity.

2.3.2 Type III-B rotaxane dendrimers. Type III-B rotaxane dendrimers refer to mechanical bonds constituted in all branching points, and generally the mechanical bond branching point is located on the threads.

The very first example of a Type III-B rotaxane dendrimer was synthesized by Vögtle et al. in 2001 through convergent synthesis. ${ }^{86}$ They began with the evaluation of the divergent approach toward rotaxane dendrimer synthesis, but failed to proceed to G1.5 rotaxane dendrimers, and thus the convergent approach was then employed. The first step was to synthesize a phenolic hydroxy-protected macrocycle with diamine and stoppered by nucleophilic substitution with 5-(benzoyloxy)isophthaloyl dichloride. [2]Rotaxane was then synthesized through the 'anion template method' with $p$-tritylphenol and 1,4-bis(bromomethyl)benzene. The rotaxane was then subjected to deprotection and generated the free phenolic hydroxy group. B'y repeating the anion template step, G1.5 [4]rotaxane dendrimer 53 was successfully synthesized (Fig. 13).

Another example of a Type III-B rotaxane dendrimer was synthesized by Qu et al. as a hetero[4]rotaxane $\mathbf{5 4}$ (Fig. 13). ${ }^{87}$ They tried both divergent and convergent approaches for the synthesis. In their convergent approaches, an azide-functionalized benzo-21-crown-7 (B21C7) crown ether was employed and [2] rotaxane was synthesized through $\mathrm{P}(n-\mathrm{Bu})_{3}$-catalyzed esterification. The azide-functionalized [2] rotaxane was reacted with DB24C8 and propargyl ended DBA through CuAAC to form a G1.5 [3] rotaxane dendrimer. In another divergent approach, it involved a self-sorting process with azide-functionalized B21C7, propargyl ended DBA and DB24C8, the propargyl ended DBA was only self-assembled with DB24C8 and a B21C7 stoppered [4] rotaxane was synthesized. The remaining two mechanical bonds were then formed by the recognition between benzylalkylammonium and $\mathrm{B} 21 \mathrm{C} 7$ followed with esterification stoppering.

Leung's (our) group reported another Type III-B G1 and G1.5 [4]rotaxane dendrimers 55 (Fig. 13) in 2013 using a convergent approach. ${ }^{73}$ We also demonstrated that it was non-cytotoxic to L929 fibroblast cells by MTT-assay and solvent-driven switching ability by a contact angle experiment. The $N$-succinimidyl ester functionalized [2] rotaxane stoppered with Fréchet dendrons was synthesized through CuAAC reaction. This functionalized [2]rotaxane was then reacted with propargyl amine to give 
A

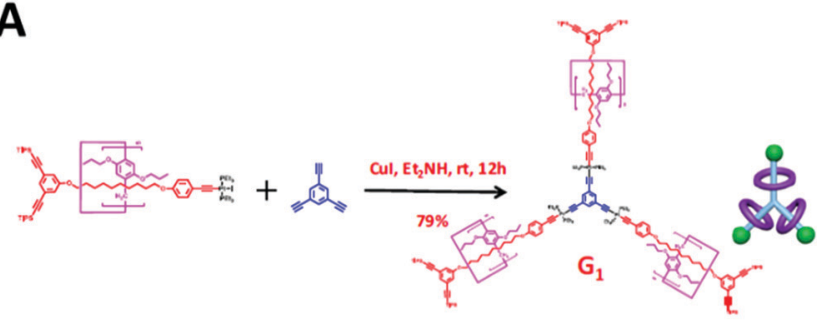

B
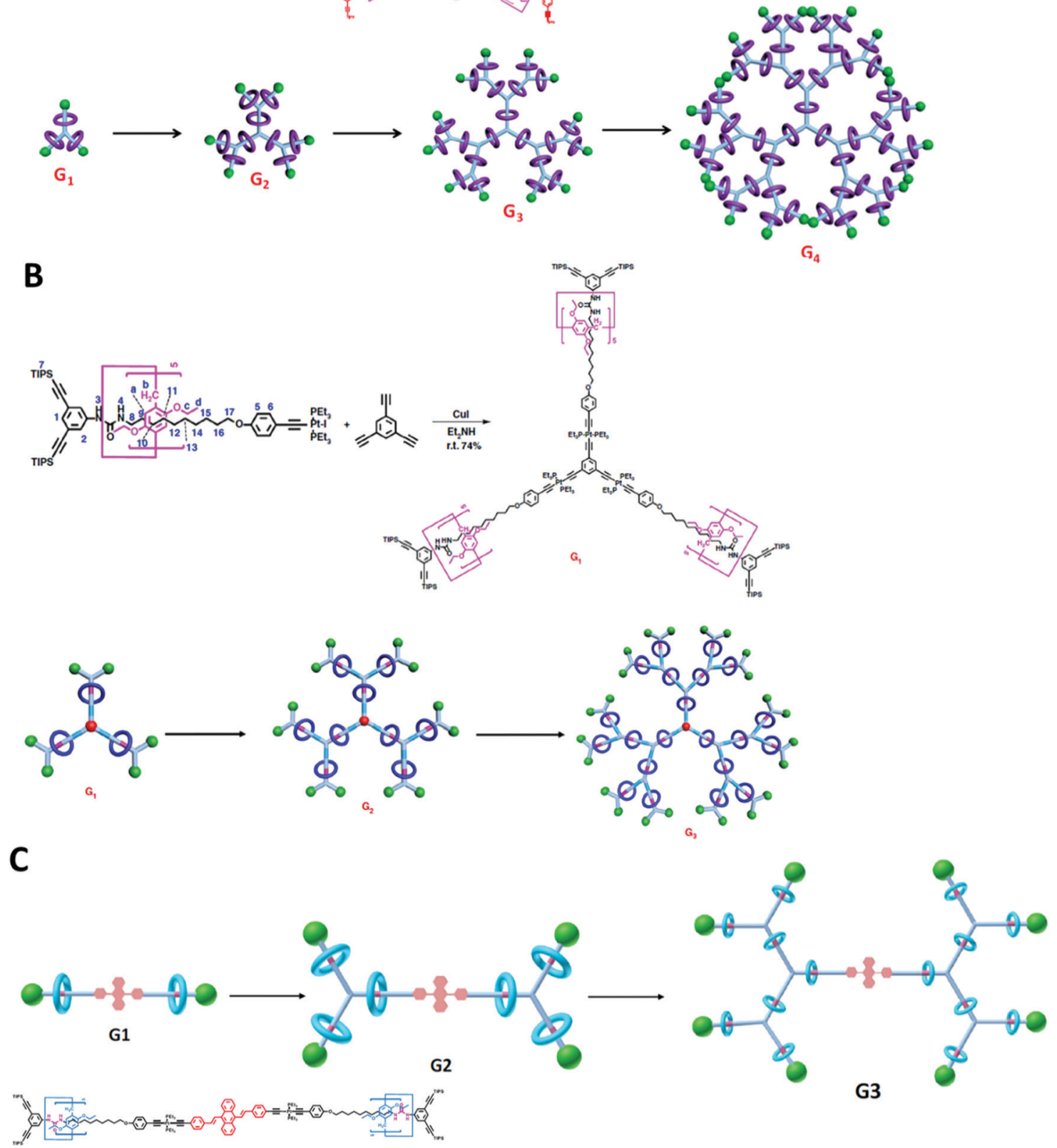

Fig. 12 Yang's Type III-A rotaxane dendrimers. (Copyright: PNAS for A, Nature Publishing group for B and Royal Society of Chemistry for C.)

propargyl functionality. Homocoupling through a Glaser-Hay reaction of [2]rotaxane itself gave a G1 [3]rotaxane dendrimer. The propargyl [2] rotaxane was reacted with one more azido ended thread and DB24C8 through CuAAC reaction gave a G1.5 [4]rotaxane dendrimer. However, the homocoupling step cannot be used for higher generation rotaxane dendrimers due to the reactivity of the Glaser-Hay reaction and steric hindrance. We then designed another synthetic approach towards higher generation rotaxane dendrimers. With the same succinimide ester functionalized [2] rotaxane, two elongated linkages with azide and acetylene were used to exchange with succinimide ester. The azido- and acetylene-functionalized rotaxane dendrons were coupled together by CuAAC reaction to form the $\mathrm{G}(n)$ rotaxane. G1 [3] rotaxane dendrimer to G3 

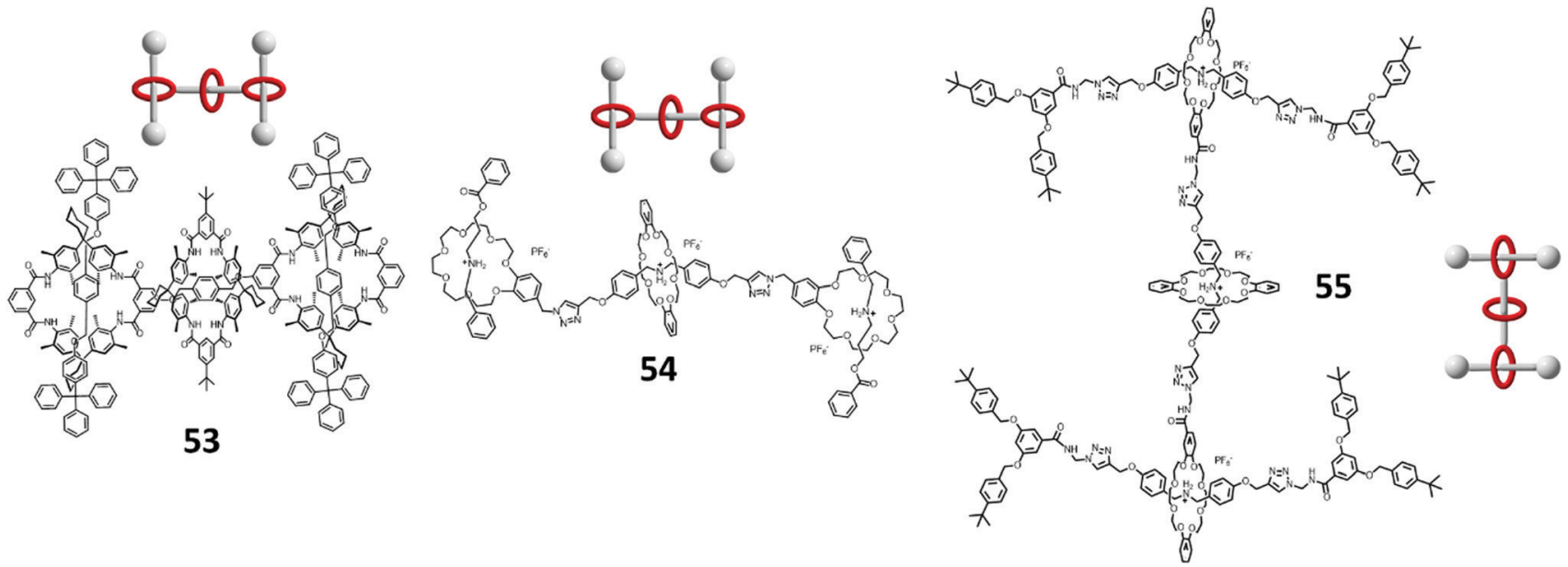

Fig. 13 Type III-B rotaxane dendrimers.

[15]rotaxane dendrimer and a G4 [16]rotaxane dendron with 15 mechanical bonds in one macromolecule were successfully synthesized (Fig. 14).$^{88}$ As the rotaxane dendrimers contained triazoles, they could act as a secondary station for DB24C8, and by deprotonating the rotaxane with phosphazene-based
BEMP resin, neutral rotaxane dendrimers were prepared. The molecular shuttling properties of different generation rotaxane dendrimers were investigated by NMR titration with 1,8-diazabicyclo[5.4.0]undec-7-ene (DBU) and TFA. The morphology of the $\mathrm{G}(n)$ rotaxane dendrimers was visualized by AFM and

A

B
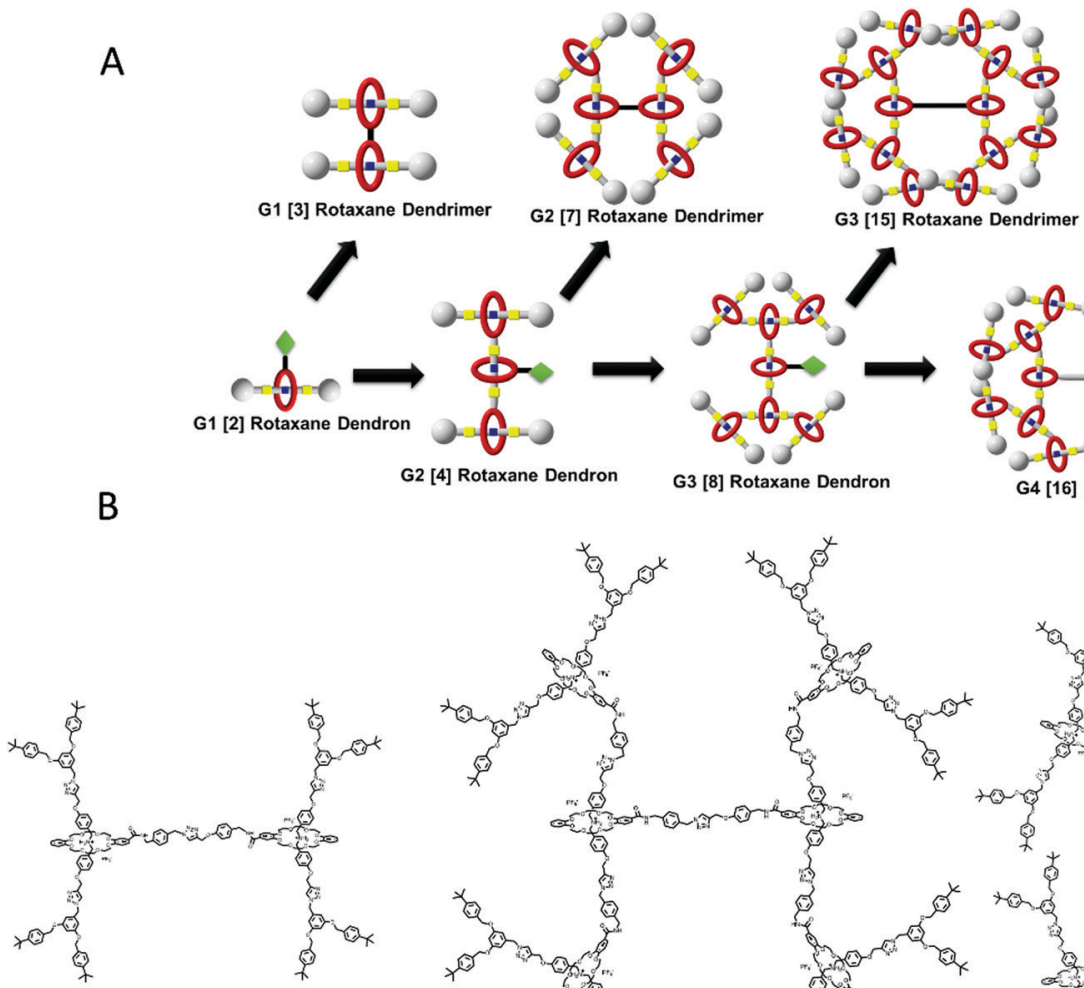

G1 [3] Rotaxane Dendrimer

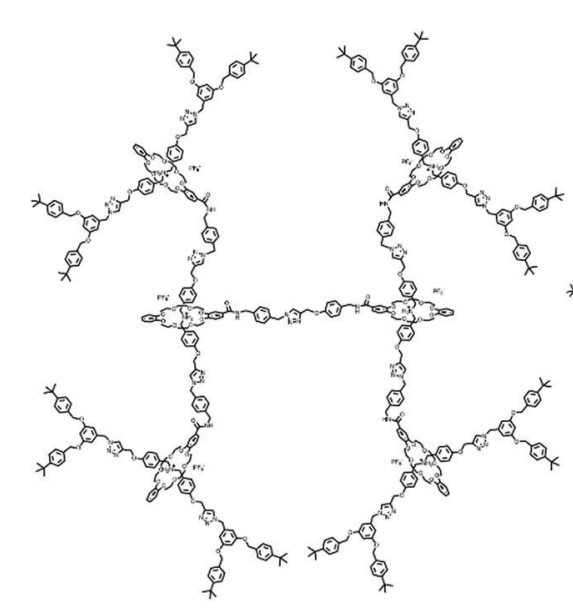

G2 [7] Rotaxane Dendrimer
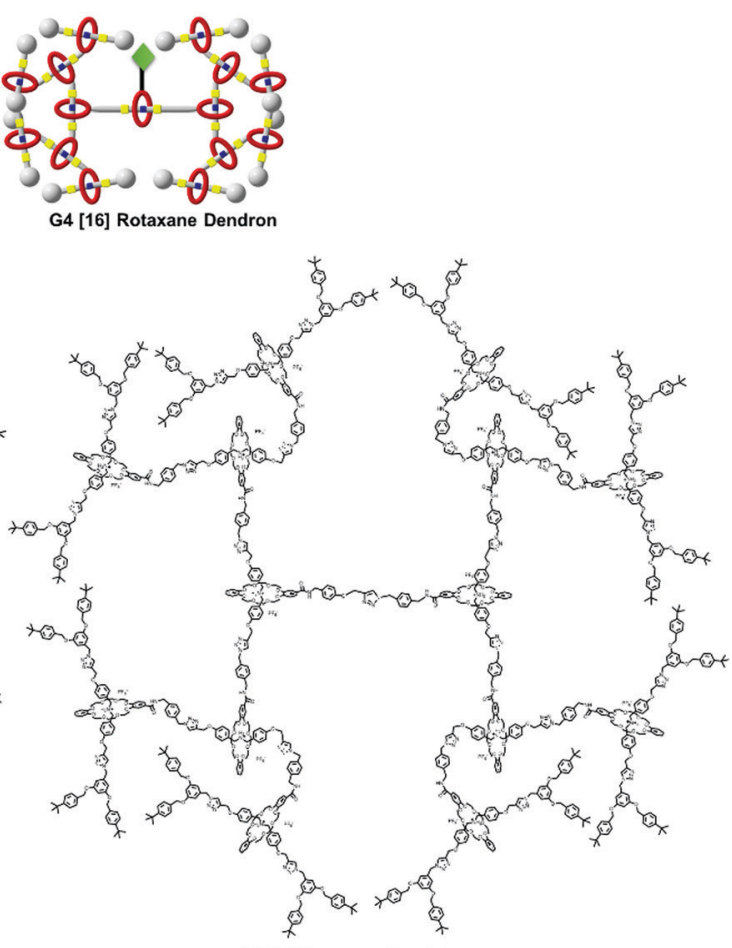

G3 [15] Rotaxane Dendrimer

Fig. 14 Leung's Type III-B rotaxane dendrimers. (Copyright: Nature Publishing Group.) 
spherical morphology were observed on a mica surface. After the deprotonation, the particle size became expanded and further confirmed by DLS, DOSY and AFM. Finally, an anticancer drug chlorambucil was used for the drug encapsulation demonstration and could be released upon addition of acid by an NMR titration experiment.

2.3.3 Type III-C rotaxane dendrimer. The Type III-C rotaxane dendrimer is the latest member of the rotaxane dendrimer family which was first categorized by Stoddart et al. in 2017. ${ }^{20}$ Type III-C rotaxane dendrimers refer to the branches extending from both rings and rods, and in general, the mechanical bonds are both in between and constitute the branching points.

The very first example of a Type III-C rotaxane dendrimer was reported by Liu et al. in 2013. First generation of Type III-C hetero [4]rotaxane dendrimers were synthesized by either using dynamic covalent chemistry 56 or olefin metathesis 57 (Fig. 15). ${ }^{89}$ A dual-functional dibenzo-26-crown-8 (DB26C8) crown ether containing two ammonium groups was synthesized. Two mechanical bonds were formed by either the dynamic clipping of 4-alkoxylsubstituted 2,6-pyridinedicarboxaldehyde with tetra(ethylene glycol) bis(2-aminophenyl)ether followed with imine reduction with $\mathrm{BH}_{3}$. THF, or bis-olefin-containing naphthalene-based pseudo-crown ether followed by ring-closing olefin metathesis. The final mechanical bonds in the middle of DB26C8 crown ether were synthesized by tributylphosphine-catalyzed esterification endcapping with 3,5-dimethylbenzoic anhydride.

In 2019, Yang et al. reported the construction of higher Type III-C rotaxane dendrimers up to fourth generation as a highly branched [46]rotaxane (Fig. 16). ${ }^{90}$ They employed the controllable divergent approach through platinum acetylide bonds with alkyne ended macrocycles and a platinum-based thread. Since the threads were composed of urea, the pillar[5] arene ring can be shuttled to the alkyl chain position upon addition of acetate anions to bind with the urea, generating an anioninduced dimension modulation. This shuttling process was reversed by the addition of $\mathrm{NaPF}_{6}$ to remove the acetate anion.
The G4 rotaxane dendrimer induced the highest dimension modulation, followed by lower generation.

Our group also reported the synthesis of up to second generation Type III-C [8]rotaxane dendrimers through a divergent approach (Fig. 17). ${ }^{91}$ Two sets of non-methylated and methylated rotaxane dendrimers performed different size modulation under the same external $\mathrm{pH}$ stimuli. The non-methylated one shrank upon the addition of a base, while the methylated one expanded. We also conducted a biological evaluation for these Type III-C rotaxane dendrimers. The in vivo experiment indicated that the dendrimers were accumulated mainly in the spleen and liver and can be removed from the mouse body completely after 48 hours. In addition, two drug molecules, chlorambucil and lithocholic acid can be bound to the dendrimers.

Type III rotaxane dendrimers are composed of the most large and complicated rotaxane architecture in comparison to Type I and II. More importantly, with the globular dimensional switching ability of these Type III rotaxane dendrimers, the prototype of 'macromolecular machines' has been achieved. Some new applications of these Type III rotaxane dendrimers as new AIE-materials and potential drug delivery carriers have been demonstrated.

\section{Operating macromolecular machines and their applications}

From all the above mentioned rotaxane dendrimers, some of them can be operated by external stimuli including $\mathrm{pH}$, redox, light, solvent and addition of anions, but most of them cannot be switched like molecular machines (Table 1). In Type I rotaxane dendrimers, the BPP34C10 in 1 can be shuttled between two $\mathrm{BIPY}^{2+}$ without control, while the molecular shuttling in 6 was governed by a redox reaction. The molecular switching of Type II rotaxane dendrimers 17, 18, 49, 50 and 51 was achieved by the addition of a base to deprotonate DBA and
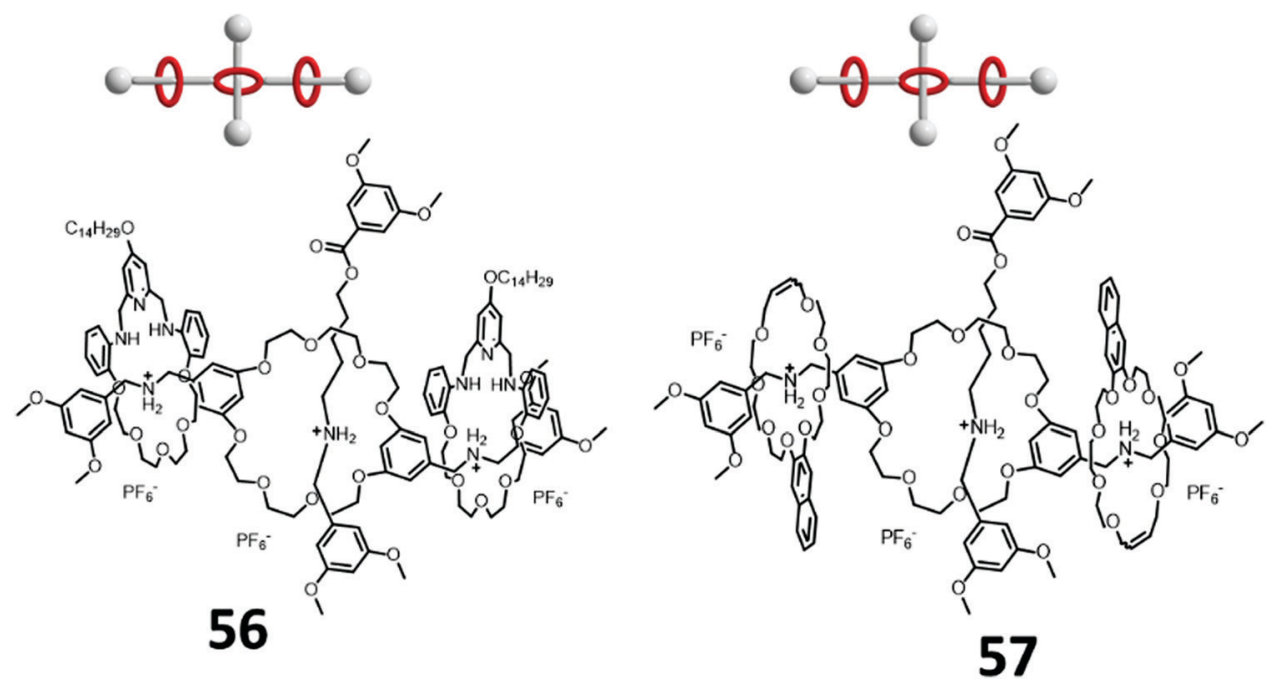

Fig. 15 Type III-C rotaxane dendrimers. 


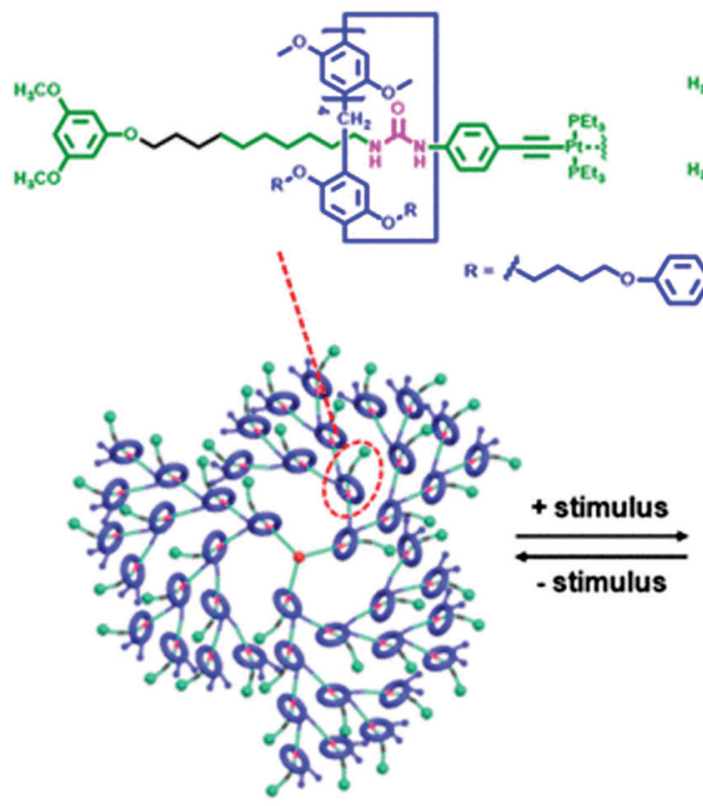

Contracted Type III-C rotaxanebranched dendrimer
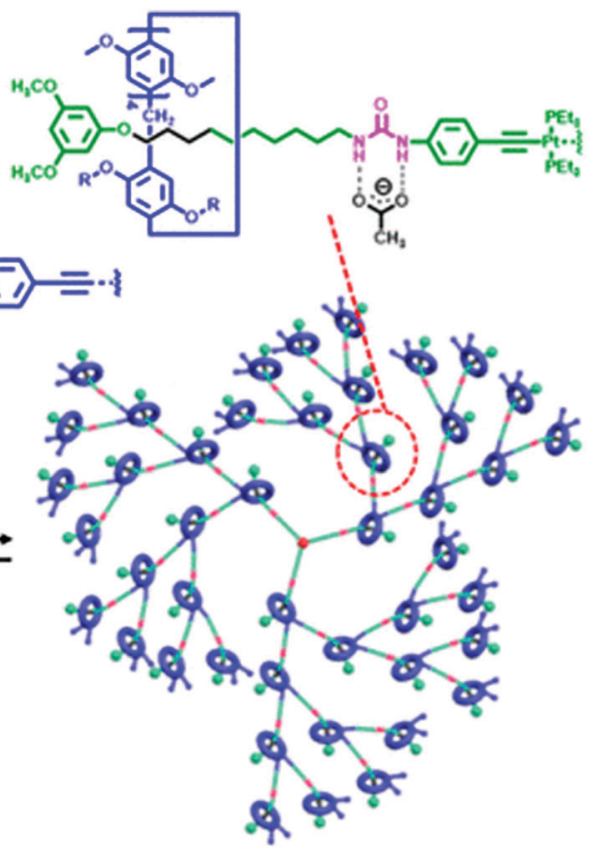

Stretched Type III-C rotaxanebranched dendrimer

Fig. 16 Yang's Type III-C rotaxane dendrimers with up to fourth generation and the anion-induced dimension modulation. (Copyright: American Chemical Society.)
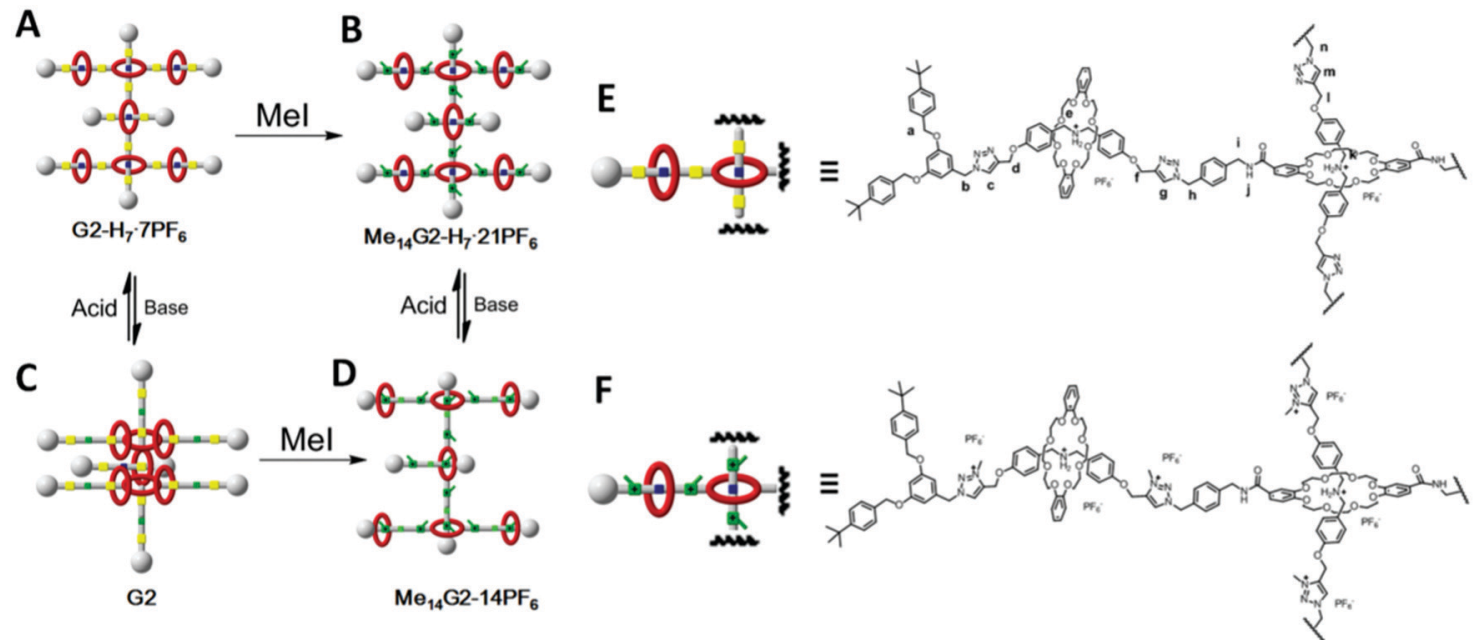

Fig. 17 Leung's non-methylated and methylated Type III-C rotaxane dendrimers. (Copyright: Royal Society of Chemistry.)

switched the DB24C8 to triazolium, and the DB24C8 were shuttled between 1,2-bis(4,4-dipyridinium)ethane in 36. Similar redox switches can also be found in Type II-A rotaxane dendrimers 22, 23 and 24 . In view of these examples, since they were all in relatively small molecular weight and first generation, only one-dimensional (1-D) single ring switching in solution and two-dimensional (2-D) small amplitude molecular operation can be achieved.

In order to achieve the macromolecular three-dimensional (3-D) modulation (multiple rings' switching in solution with multiple directions), higher generation of Type III macromolecular machines were dissolved in the solution. The first demonstration of such 3-D macromolecular size modulation was achieved by higher generation Type III-B rotaxane dendrimers reported by our group in $2018,{ }^{88}$ where these macromolecular machines were operated by $\mathrm{pH}$. The void space of such macromolecular machines can be easily tuned by acids, which cannot be achieved by normal dendrimers and simple molecular machines. Yang's group later reported such macromolecular machines operation by solvent and anions with up to G4 Type III-A rotaxane dendrimers. ${ }^{84}$ More recently, Yang's reported another new Type III-C rotaxane dendrimer operated 
Table 1 Summary of each switchable rotaxane dendrimer

\begin{tabular}{|c|c|c|c|c|}
\hline Motion & $\begin{array}{l}\text { Dendrimer } \\
\text { type }\end{array}$ & $\begin{array}{l}\text { Switching } \\
\text { stimuli }\end{array}$ & $\begin{array}{l}\text { \% change } \\
\text { (characterization method) }\end{array}$ & Ref. \\
\hline \multirow[t]{2}{*}{ 1-Dimensional (single ring switching in solution) } & $\mathrm{I}-\mathrm{A}, \mathrm{I}-\mathrm{C}$ & Auto-switching & $\mathrm{N} / \mathrm{A}$ & 21 and 23 \\
\hline & $\mathrm{I}-\mathrm{A}$ & Redox & N/A & 27 \\
\hline \multirow{4}{*}{$\begin{array}{l}\text { 2-Dimensional (multiple rings' switching in solution } \\
\text { with one direction) }\end{array}$} & II-A & $\mathrm{pH}$ & N/A & 45 and 46 \\
\hline & II-A & Redox & N/A & $50-52$ \\
\hline & II-B & $\mathrm{pH}$ & N/A & 73 \\
\hline & II-C & $\mathrm{pH}$ & N/A & 80 and 81 \\
\hline \multirow{5}{*}{$\begin{array}{l}\text { 3-Dimensional (multiple rings' switching in solution } \\
\text { with multiple directions) }\end{array}$} & III-A & Anion & 29-43\% (DOSY) & 84 \\
\hline & III-B & Solvent & $26 \%$ (contact angle) & 73 \\
\hline & III-B & $\mathrm{pH}$ & $20-22 \%(\mathrm{AFM})$ & 88 \\
\hline & III-C & Anion & 20-35\% (DOSY) & 90 \\
\hline & III-C & $\mathrm{pH}$ & 8-21\% (AFM) & 91 \\
\hline
\end{tabular}

by anions and showed a larger 3-D size modulation. ${ }^{90}$ Our group described that Type III-C rotaxane dendrimers can have two different modulation modes under the same external stimuli with the methylated rotaxane dendrimers. ${ }^{91}$ The drug binding ability of such macromolecular machines was shown, and it was non-toxic in a mouse physiological environment. ${ }^{73,88,91}$ The development of such macromolecular machines with fascinating macromolecular operation is unlimited. For a very recent example, Yang's group developed new elegant 'daisy chain dendrimers' which could open a new page in the field. ${ }^{92}$ It is believed that this new class of rotaxane dendrimer material could finally be utilized in various advanced materials.

\section{Conclusion and outlook}

In conclusion, throughout the past few decades, molecular mechanists are trying to explore the complicated rotaxane dendrimer structures, from simple dendron grafted Type I rotaxane dendrimers to hyperbranched dendritic Type III polyrotaxane dendrimer macromolecules. The synthetic challenges of preparing Type I and Type II rotaxane dendrimers have been solved by chemists using various approaches. In Type II rotaxane dendrimers, there is still room for chemists to prepare a core generation larger than two rotaxane dendrimers, such that the surface could be accommodating many more mechanical bonds. The synthesis of Type III polyrotaxane dendrimers is still a very challenging task for synthetic chemists, especially for the facile and efficient preparation of higher generation hyperbranched rotaxane dendrimers. Many more types of a rotaxane dendrimer family member can also be categorized by chemists, providing infinite opportunity for creative and elegant new members into the rotaxane dendrimer family, More importantly, nearly all examples described in this review were only focused on the design and synthesis of different rotaxane dendrimers but lack actual applications. The next step in this field, however, will be finding the fascinating intrinsic properties of rotaxane dendrimers and applying them in various disciplines such as drug delivery, catalysis, molecular electronics etc.

\section{Conflicts of interest}

There are no conflicts to declare.

\section{Acknowledgements}

K. C.-F. Leung acknowledges the President's Award for Outstanding Performance in Research Supervision and C.-S. Kwan acknowledges the Dr Wu Yee Sun Memorial Scholarship from The Hong Kong Baptist University (HKBU). This work was partially supported by The State Key Laboratory of Environmental and Biological Analysis, HKBU (SKLP-1920-P05).

\section{References}

1 E. Buhleier, W. Wehner and F. Vögtle, "Cascade"- and "Nonskid-Chain-like" Syntheses of Molecular Cavity Topologies, Synthesis, 1978, 155-158.

2 D. A. Tomalia, H. Baker, J. Dewald, M. Hall, G. Kallos, S. Martin, J. Roeck, J. Ryder and P. Smith, A New Class of Polymers: Starburst-Dendritic Macromolecules, Polym. J., 1985, 17, 117-132.

3 S. M. Grayson and J. M. J. Fréchet, Convergent Dendrons and Dendrimers: from Synthesis to Applications, Chem. Rev., 2001, 101, 3819-3868.

4 C. J. Hawker and J. M. J. Fréchet, Preparation of polymers with controlled molecular architecture. A new convergent approach to dendritic macromolecules, J. Am. Chem. Soc., 1990, 112, 7638-7647.

5 D. Astruc, E. Boisselier and C. Ornelas, Dendrimers Designed for Functions: From Physical, Photophysical, and Supramolecular Properties to Applications in Sensing, Catalysis, Molecular Electronics, Photonics, and Nanomedicine, Chem. Rev., 2010, 110, 1857-1959.

6 U. Boas and P. M. H. Heegaard, Dendrimers in drug research, Chem. Soc. Rev., 2004, 33, 43-63.

7 E. R. Gillies and J. M. J. Fréchet, Dendrimers and dendritic polymers in drug delivery, Drug Discovery Today, 2005, 10, 35-43.

8 V. S. Myers, M. G. Weir, E. V. Carino, D. F. Yancey, S. Pande and R. M. Crooks, Dendrimer-encapsulated nanoparticles: new synthetic and characterization methods and catalytic applications, Chem. Sci., 2011, 2, 1632-1646.

9 D. Astruc and F. Chardac, Dendritic Catalysts and Dendrimers in Catalysis, Chem. Rev., 2001, 101, 2991-3024.

10 A. Adronov and J. M. J. Fréchet, Light-harvesting dendrimers, Chem. Commun., 2000, 1701-1710. 
11 D. B. Amabilino, M. Asakawa, P. R. Ashton, R. Ballardini, V. Balzani, M. Bělohradský, A. Credi, M. Higuchi, F. M. Raymo, T. Shimizu, J. F. Stoddart, M. Venturi and K. Yase, Aggregation of self-assembling branched [n]rotaxanes, New J. Chem., 1998, 22, 959-972.

12 B. Donnio, S. Buathong, I. Bury and D. Guillon, Liquid crystalline dendrimers, Chem. Soc. Rev., 2007, 36, 1495-1513.

13 J.-P. Sauvage, From Chemical Topology to Molecular Machines (Nobel Lecture), Angew. Chem., Int. Ed., 2017, 56, 11080-11093.

14 J. F. Stoddart, Mechanically Interlocked Molecules (MIMs)Molecular Shuttles, Switches, and Machines (Nobel Lecture), Angew. Chem., Int. Ed., 2017, 56, 11094-11125.

15 H. Tian and Q.-C. Wang, Recent progress on switchable rotaxanes, Chem. Soc. Rev., 2006, 35, 361-374.

16 M. Xue, Y. Yang, X. Chi, X. Yan and F. Huang, Development of Pseudorotaxanes and Rotaxanes: From Synthesis to Stimuli-Responsive Motions to Applications, Chem. Rev., 2015, 115, 7398-7501.

17 S. Erbas-Cakmak, D. A. Leigh, C. T. McTernan and A. L. Nussbaumer, Artificial Molecular Machines, Chem. Rev., 2015, 115, 10081-10206.

18 J. Lee and K. Kim, Rotaxane dendrimers, Top. Curr. Chem., 2003, 228, 111-140.

19 K. C.-F. Leung and K.-N. Lau, Self-assembly and thermodynamic synthesis of rotaxane dendrimers and related structures, Polym. Chem., 2010, 1, 988-1000.

20 C. J. Bruns and J. F. Stoddart, The nature of the mechanical bond: from molecules to machines, John Wiley \& Sons, 2016.

21 D. B. Amabilino, P. R. Ashton, V. Balzani, C. L. Brown, A. Credi, J. M. J. Fréchet, J. W. Leon, F. M. Raymo, N. Spencer, J. F. Stoddart and M. Venturi, Self-Assembly of [n]Rotaxanes Bearing Dendritic Stoppers, J. Am. Chem. Soc., 1996, 118, 12012-12020.

22 G. M. Hübner, G. Nachtsheim, Q. Y. Li, C. Seel and F. Vögtle, The Spatial Demand of Dendrimers: Deslipping of Rotaxanes, Angew. Chem., Int. Ed., 2000, 39, 1269-1272.

23 D. A. Tramontozzi, N. D. Suhan, S. H. Eichhorn and S. J. Loeb, The Effect of Incorporating Fréchet Dendrons into Rotaxanes and Molecular Shuttles Containing the 1,2-Bis(pyridinium)ethane $\subset[24]$ Crown-8 Templating Motif, Chem. - Eur. J., 2010, 16, 4466-4476.

24 G. Liu, Z. Li, D. Wu, W. Xue, T. Li, S. H. Liu and J. Yin, Dendritic [2]Rotaxanes: Synthesis, Characterization, and Properties, J. Org. Chem., 2014, 79, 643-652.

25 M. Feng, X. Guo, X. Lin, X. He, W. Ji, S. Du, D. Zhang, D. Zhu and H. Gao, Stable, Reproducible Nanorecording on Rotaxane Thin Films, J. Am. Chem. Soc., 2005, 127, 15338-15339.

26 W.-K. Wang, Z.-Y. Xu, Y.-C. Zhang, H. Wang, D.-W. Zhang, Y. Liu and Z.-T. Li, A tristable [2] rotaxane that is doubly gated by foldamer and azobenzene kinetic barriers, Chem. Commun., 2016, 52, 7490-7493.

27 I. Aprahamian, T. Yasuda, T. Ikeda, S. Saha, W. R. Dichtel, K. Isoda, T. Kato and J. F. Stoddart, A Liquid-Crystalline Bistable [2]Rotaxane, Angew. Chem., Int. Ed., 2007, 46, 4675-4679.
28 S. C. Han, J. Yoon, J. Oh and J. W. Lee, Synthesis of [3]-rotaxane dendrimers by host-mediated click chemistry, Bull. Korean Chem. Soc., 2011, 32, 3809-3812.

29 J.-W. Lee, S.-C. Han, J.-H. Kim, Y.-H. Ko and K.-M. Kim, Formation of rotaxane dendrimers by supramolecular click chemistry, Bull. Korean Chem. Soc., 2007, 28, 1837-1840.

30 S. Xiao, N. Fu, K. Peckham and B. D. Smith, Efficient Synthesis of Fluorescent Squaraine Rotaxane Dendrimers, Org. Lett., 2010, 12, 140-143.

31 B. Kenda and F. Diederich, Supramolecular Aggregates of Dendritic Cyclophanes (Dendrophanes) Threaded on Molecular Rods with Steroid Termini, Angew. Chem., Int. Ed., 1998, 37, 3154-3158.

32 H. W. Gibson, N. Yamaguchi, L. Hamilton and J. W. Jones, Cooperative Self-Assembly of Dendrimers via Pseudorotaxane Formation from a Homotritopic Guest Molecule and Complementary Monotopic Host Dendrons, J. Am. Chem. Soc., 2002, 124, 4653-4665.

33 K. C.-F. Leung, F. Aricó, S. J. Cantrill and J. F. Stoddart, Template-Directed Dynamic Synthesis of Mechanically Interlocked Dendrimers, J. Am. Chem. Soc., 2005, 127, 5808-5810.

34 K. C.-F. Leung, F. Aricó, S. J. Cantrill and J. F. Stoddart, Dynamic Mechanically Interlocked Dendrimers: Amplification in Dendritic Dynamic Combinatorial Libraries, Macromolecules, 2007, 40, 3951-3959.

35 G. R. Newkome, Molecular recognition using $\beta$-cyclodextrinmodified dendrimers: novel building blocks for convergent self-assembly, Chem. Commun., 1998, 1821-1822.

36 Q. Li, K. Han, J. Li, X. Jia and C. Li, Synthesis of dendrimerfunctionalized pillar[5]arenes and their self-assembly to dimeric and trimeric complexes, Tetrahedron Lett., 2015, 56, 3826-3829.

37 C. Reuter, G. Pawlitzki, U. Wörsdörfer, M. Plevoets, A. Mohry, T. Kubota, Y. Okamoto and F. Vögtle, Chiral Dendrophanes, Dendro[2]rotaxanes, and Dendro[2]catenanes: Synthesis and Chiroptical Phenomena, Eur. J. Org. Chem., 2000, 3059-3067.

38 A. M. Elizarov, T. Chang, S.-H. Chiu and J. F. Stoddart, SelfAssembly of Dendrimers by Slippage, Org. Lett., 2002, 4, 3565-3568.

39 K.-S. Jeong and E.-J. Park, Self-Assembly of Interlocked Supramolecular Dendrimers, J. Org. Chem., 2004, 69, 2618-2621.

40 Z.-Q. Cao, Y.-C. Wang, A.-H. Zou, G. London, Q. Zhang, C. Gao and D.-H. Qu, Reversible switching of a supramolecular morphology driven by an amphiphilic bistable [2]rotaxane, Chem. Commun., 2017, 53, 8683-8686.

41 R. Castro, I. Cuadrado, B. Alonso, C. M. Casado, M. Morán and A. E. Kaifer, Multisite Inclusion Complexation of Redox Active Dendrimer Guests, J. Am. Chem. Soc., 1997, 119, 5760-5761.

42 B. González, C. M. Casado, B. Alonso, I. Cuadrado, M. Morán, Y. Wang and A. E. Kaifer, Synthesis, electrochemistry and cyclodextrin binding of novel cobaltocenium-functionalized dendrimers, Chem. Commun., 1998, 2569-2570.

43 M. W. P. L. Baars, A. J. Karlsson, V. Sorokin, B. F. W. de Waal and E. W. Meijer, Supramolecular Modification of 
the Periphery of Dendrimers Resulting in Rigidity and Functionality, Angew. Chem., Int. Ed., 2000, 39, 4262-4265.

44 J. W. Lee, Y. H. Ko, S.-H. Park, K. Yamaguchi and K. Kim, Novel Pseudorotaxane-Terminated Dendrimers: Supramolecular Modification of Dendrimer Periphery, Angew. Chem., Int. Ed., 2001, 40, 746-749.

45 J.-N. Zhang, H. Li, W. Zhou, S.-L. Yu, D.-H. Qu and H. Tian, Fluorescence Modulation in Tribranched Switchable [4]Rotaxanes, Chem. - Eur. J., 2013, 19, 17192-17200.

46 H. Zhang, Q. Liu, J. Li and D.-H. Qu, A Novel Star-Shaped Zinc Porphyrin Cored [5]Rotaxane, Org. Lett., 2013, 15, 338-341.

47 G. Yu, Y. Suzaki and K. Osakada, Ferrocene-containing [1]-, [2]-, [3]- and [4] rotaxanes synthesized from a common precursor, $R S C A d v .$, 2016, 6, 41369-41375.

48 T. H. Ngo, J. Labuta, G. N. Lim, W. A. Webre, F. D’Souza, P. A. Karr, J. E. M. Lewis, J. P. Hill, K. Ariga and S. M. Goldup, Porphyrinoid rotaxanes: building a mechanical picket fence, Chem. Sci., 2017, 8, 6679-6685.

49 J. Lewis, J. Winn and S. Goldup, Stepwise, protecting group free synthesis of [4] rotaxanes, Molecules, 2017, 22, 89.

50 D. B. Amabilino, P. R. Ashton, M. Bělohradský, F. M. Raymo and J. F. Stoddart, The self-assembly of branched $[n]$ rotaxanes-the first step towards dendritic rotaxanes, Chem. Commun., 1995, 751-753.

51 I. Aprahamian, J.-C. Olsen, A. Trabolsi and J. F. Stoddart, Tetrathiafulvalene Radical Cation Dimerization in a Bistable Tripodal [4]Rotaxane, Chem. - Eur. J., 2008, 14, 3889-3895.

52 A. B. Braunschweig, W. R. Dichtel, O. Š. Miljanić, M. A. Olson, J. M. Spruell, S. I. Khan, J. R. Heath and J. F. Stoddart, Modular Synthesis and Dynamics of a Variety of Donor-Acceptor Interlocked Compounds Prepared by Click Chemistry, Chem. - Asian J., 2007, 2, 634-647.

53 F. Aricó, T. Chang, S. J. Cantrill, S. I. Khan and J. F. Stoddart, Template-Directed Synthesis of Multiply Mechanically Interlocked Molecules Under Thermodynamic Control, Chem. - Eur. J., 2005, 11, 4655-4666.

54 D. Tuncel and J. H. G. Steinke, The synthesis of [2], [3] and [4] rotaxanes and semirotaxanes, Chem. Commun., 2002, 496-497.

55 M. Özkan, Y. Keser, S. E. Hadi and D. Tuncel, A [5]RotaxaneBased Photosensitizer for Photodynamic Therapy, Eur. J. Org. Chem., 2019, 3534-3541.

56 M. He, L. Chen, B. Jiang, H. Tan, C. Wang and H. Yang, Facile construction of $\mathrm{Zn}$ (II)-porphyrin-cored [5]rotaxane and its controllable aggregation behaviours, Chin. Chem. Lett., 2019, 30, 131-134.

57 Y. Tokunaga, T. Ito, H. Sugawara and R. Nakata, Dynamic covalent chemistry of a boronylammonium ion and a crown ether: formation of a C3-symmetric [4]rotaxane, Tetrahedron Lett., 2008, 49, 3449-3452.

58 H. Sasabe, N. Kihara, K. Mizuno, A. Ogawa and T. Takata, Efficient synthesis of [2]- and higher order rotaxanes via the transition metal-catalyzed hydrosilylation of alkyne, Tetrahedron Lett., 2005, 46, 3851-3853.
59 A. Fernandez, J. Ferrando-Soria, E. M. Pineda, F. Tuna, I. J. Vitorica-Yrezabal, C. Knappke, J. Ujma, C. A. Muryn, G. A. Timco and P. E. Barran, Making hybrid [n]-rotaxanes as supramolecular arrays of molecular electron spin qubits, Nat. Commun., 2016, 7, 10240.

60 M. Mao, X.-K. Zhang, T.-Y. Xu, X.-D. Wang, S.-J. Rao, Y. Liu, D.-H. Qu and H. Tian, Towards a hexa-branched [7]rotaxane from a [3]rotaxane via a $[2+2+2]$ alkyne cyclotrimerization process, Chem. Commun., 2019, 55, 3525-3528.

61 W. Wang, B. Sun, X.-Q. Wang, Y.-Y. Ren, L.-J. Chen, J. Ma, Y. Zhang, X. Li, Y. Yu, H. Tan and H.-B. Yang, Discrete Stimuli-Responsive Multirotaxanes with Supramolecular Cores Constructed through a Modular Approach, Chem. Eur. J., 2015, 21, 6286-6294.

62 Y.-X. Wang, Q.-F. Zhou, L.-J. Chen, L. Xu, C.-H. Wang, X. Li and H.-B. Yang, Facile construction of organometallic rotaxaneterminated dendrimers using neutral platinum-acetylides as the main scaffold, Chem. Commun., 2018, 54, 2224-2227.

63 P. R. Ashton, J. A. Preece, J. F. Stoddart and M. S. Tolley, Template-Directed Syntheses of a Bis[2]catenane and a Bis[2]rotaxane-Towards Self-Assembling Polymers, Synlett, 1994, 789-792.

64 P. R. Ashton, J. Huff, S. Menzer, I. W. Parsons, J. A. Preece, J. F. Stoddart, M. S. Tolley, A. J. P. White and D. J. Williams, Bis[2]catenanes and a bis[2]rotaxane-Model Compounds for Polymers with Mechanically Interlocked Components, Chem. - Eur. J., 1996, 2, 31-44.

65 F. Aricó, T. Chang, S. J. Cantrill, S. I. Khan and J. F. Stoddart, Template-Directed Synthesis of Multiply Mechanically Interlocked Molecules Under Thermodynamic Control, Chem. - Eur. J., 2005, 11, 4655-4666.

66 A. M. Elizarov, S.-H. Chiu, P. T. Glink and J. F. Stoddart, Dendrimer with Rotaxane-Like Mechanical Branching, Org. Lett., 2002, 4, 679-682.

67 S. J. Loeb and D. A. Tramontozzi, Branched $[n]$ rotaxanes ( $n=2-4)$ from multiple dibenzo-24-crown-8 ether wheels and 1,2-bis(4,4'-dipyridinium)ethane axles, Org. Biomol. Chem., 2005, 3, 1393-1401.

68 W. Zhou, Y.-J. Guo and D.-H. Qu, Photodriven Clamlike Motion in a [3]Rotaxane with Two [2]Rotaxane Arms Bridged by an Overcrowded Alkene Switch, J. Org. Chem., 2013, 78, 590-596.

69 M. Narita, I. Yoon, M. Aoyagi, M. Goto, T. Shimizu and M. Asakawa, Transition Metal(II)-Salen and -Salophen Macrocyclic Complexes for Rotaxane Formation: Syntheses and Crystal Structures, Eur. J. Inorg. Chem., 2007, 4229-4237.

70 A. Noor, S. C. Moratti and J. D. Crowley, Active-template synthesis of "click" [2]rotaxane ligands: self-assembly of mechanically interlocked metallo-supramolecular dimers, macrocycles and oligomers, Chem. Sci., 2014, 5, 4283-4290.

71 D. R. Kohn, L. D. Movsisyan, A. L. Thompson and H. L. Anderson, Porphyrin-Polyyne [3]- and [5]Rotaxanes, Org. Lett., 2017, 19, 348-351.

72 M. Franz, J. A. Januszewski, F. Hampel and R. R. Tykwinski, [3]Rotaxanes with Mixed Axles: Polyynes and Cumulenes, Eur. J. Org. Chem., 2019, 3503-3512. 
73 W. K. W. Ho, S.-F. Lee, C.-H. Wong, X.-M. Zhu, C.-S. Kwan, C.-P. Chak, P. M. Mendes, C. H. K. Cheng and K. C.-F. Leung, Type III-B rotaxane dendrimers, Chem. Commun., 2013, 49, 10781-10783.

74 H.-B. Yang, K. Ghosh, B. H. Northrop, Y.-R. Zheng, M. M. Lyndon, D. C. Muddiman and P. J. Stang, A Highly Efficient Approach to the Self-Assembly of Hexagonal CavityCored Tris[2]pseudorotaxanes from Several Components via Multiple Noncovalent Interactions, J. Am. Chem. Soc., 2007, 129, 14187-14189.

75 X.-D. Xu, H.-B. Yang, Y.-R. Zheng, K. Ghosh, M. M. Lyndon, D. C. Muddiman and P. J. Stang, Self-Assembly of Dendritic Tris(crown ether) Hexagons and Their Complexation with Dibenzylammonium Cations, J. Org. Chem., 2010, 75, 7373-7380.

76 X.-D. Xu, J.-K. Ou-Yang, J. Zhang, Y.-Y. Zhang, H.-Y. Gong, Y. Yu and H.-B. Yang, Self-assembly of supramolecular tris(crown ether) hexagons with dendritic dibenzylammonium cations, Tetrahedron, 2013, 69, 1086-1091.

77 A. Mohry, H. Schwierz and F. Vögtle, Supramolecular assemblies: a bis(pretzelane) and a tetrakis(rotaxane), Synthesis, 1999, 1753-1758.

78 J. W. Jones, W. S. Bryant, A. W. Bosman, R. A. J. Janssen, E. W. Meijer and H. W. Gibson, Crowned Dendrimers: pH-Responsive Pseudorotaxane Formation, J. Org. Chem., 2003, 68, 2385-2389.

79 S. K. Dey, F. Beuerle, M. A. Olson and J. F. Stoddart, Arranging pseudorotaxanes octahedrally around [60]fullerene, Chem. Commun., 2011, 47, 1425-1427.

80 H. Li, X. Li, H. Ågren and D.-H. Qu, Two Switchable Star-Shaped [1](n)Rotaxanes with Different Multibranched Cores, Org. Lett., 2014, 16, 4940-4943.

81 P. Waelès, C. Clavel, K. Fournel-Marotte and F. Coutrot, Synthesis of triazolium-based mono- and tris-branched [1] rotaxanes using a molecular transporter of dibenzo-24crown-8, Chem. Sci., 2015, 6, 4828-4836.

82 S.-Y. Kim, Y. H. Ko, J. W. Lee, S. Sakamoto, K. Yamaguchi and K. Kim, Toward High-Generation Rotaxane Dendrimers That Incorporate a Ring Component on Every Branch: Noncovalent Synthesis of a Dendritic [10]Pseudorotaxane with 13 Molecular Components, Chem. - Asian J., 2007, 2, 747-754.
83 W. Wang, L.-J. Chen, X.-Q. Wang, B. Sun, X. Li, Y. Zhang, J. Shi, Y. Yu, L. Zhang, M. Liu and H.-B. Yang, Organometallic rotaxane dendrimers with fourth-generation mechanically interlocked branches, Proc. Natl. Acad. Sci. U. S. A., 2015, 112, 5597-5601.

84 X.-Q. Wang, W. Wang, W.-J. Li, L.-J. Chen, R. Yao, G.-Q. Yin, Y.-X. Wang, Y. Zhang, J. Huang, H. Tan, Y. Yu, X. Li, L. Xu and H.-B. Yang, Dual stimuli-responsive rotaxane-branched dendrimers with reversible dimension modulation, Nat. Commun., 2018, 9, 3190.

85 X.-Q. Wang, W. Wang, W.-J. Li, Y. Qin, G.-Q. Yin, W.-L. Jiang, X. Li, S. Wu and H.-B. Yang, Rotaxane-branched dendrimers with aggregation-induced emission behavior, Org. Chem. Front., 2019, 6, 1686-1691.

86 F. Osswald, E. Vogel, O. Safarowsky, F. Schwanke and F. Vögtle, Rotaxane assemblies with dendritic architecture, Adv. Synth. Catal., 2001, 343, 303-309.

87 Q.-F. Luo, L. Zhu, S.-J. Rao, H. Li, Q. Miao and D.-H. Qu, Two Stepwise Synthetic Routes toward a Hetero[4]rotaxane, J. Org. Chem., 2015, 80, 4704-4709.

88 C.-S. Kwan, R. Zhao, M. A. Van Hove, Z. Cai and K. C.-F. Leung, Higher-generation type III-B rotaxane dendrimers with controlling particle size in three-dimensional molecular switching, Nat. Commun., 2018, 9, 497.

89 Z. Li, G. Liu, W. Xue, D. Wu, Y.-W. Yang, J. Wu, S. H. Liu, J. Yoon and J. Yin, Construction of Hetero $[n]$ rotaxanes by Use of Polyfunctional Rotaxane Frameworks, J. Org. Chem., 2013, 78, 11560-11570.

90 X.-Q. Wang, W.-J. Li, W. Wang, J. Wen, Y. Zhang, H. Tan and H.-B. Yang, Construction of Type III-C Rotaxane-Branched Dendrimers and Their Anion-Induced Dimension Modulation Feature, J. Am. Chem. Soc., 2019, 141, 13923-13930.

91 C.-S. Kwan, T. Wang, M. Li, A. S. C. Chan, Z. Cai and K. C.-F. Leung, Type III-C rotaxane dendrimers: synthesis, dual size modulation and in vivo evaluation, Chem. Commun., 2019, 55, 13426-13429.

92 W.-J. Li, W. Wang, X.-Q. Wang, M. Li, Y. Ke, R. Yao, J. Wen, G.-Q. Yin, B. Jiang, X. Li, P. Yin and H.-B. Yang, Daisy Chain Dendrimers: Integrated Mechanically Interlocked Molecules with Stimuli-Induced Dimension Modulation Feature, J. Am. Chem. Soc., 2020, 142, 8473-8482. 